\title{
Optogenetic Stimulation of the Superior Colliculus Confers Retinal Neuroprotection in a Mouse Glaucoma Model
}

\author{
Emiel Geeraerts, ${ }^{1,2}$ Marie Claes, ${ }^{1,2}$ Eline Dekeyster, ${ }^{1,2}$ Manuel Salinas-Navarro, ${ }^{1,2}$ Lies De Groef, ${ }^{1,2}$ \\ Chris Van den Haute, ${ }^{2,3,4}$ Isabelle Scheyltjens, ${ }^{2,5}$ Veerle Baekelandt, ${ }^{2,3}$ Lutgarde Arckens, ${ }^{2,5}$ and Lieve Moons ${ }^{1,2}$ \\ ${ }^{1}$ Laboratory of Neural Circuit Development and Regeneration, Department of Biology, KU Leuven, 3000 Leuven, Belgium, ${ }^{2}$ Leuven Brain Institute, KU \\ Leuven, 3000 Leuven, Belgium, ${ }^{3}$ Laboratory for Neurobiology and Gene Therapy, Department of Neurosciences, KU Leuven, 3000 Leuven, Belgium, ${ }^{4}$ Viral \\ Vector Core Leuven, KU Leuven, 3000 Leuven, Belgium, and ${ }^{5}$ Laboratory of Neuroplasticity and Neuroproteomics, Department of Biology; KU Leuven, \\ 3000 Leuven, Belgium
}

Glaucoma is characterized by a progressive loss of retinal ganglion cells (RGCs) in the eye, which ultimately results in visual impairment or even blindness. Because current therapies often fail to halt disease progression, there is an unmet need for novel neuroprotective therapies to support RGC survival. Various research lines suggest that visual target centers in the brain support RGC functioning and survival. Here, we explored whether increasing neuronal activity in one of these projection areas could improve survival of RGCs in a mouse glaucoma model. Prolonged activation of an important murine RGC target area, the superior colliculus (SC), was established via a novel optogenetic stimulation paradigm. By leveraging the unique channel kinetics of the stabilized step function opsin (SSF0), protracted stimulation of the SC was achieved with only a brief light pulse. SSFO-mediated collicular stimulation was confirmed by immunohistochemistry for the immediate-early gene c-Fos and behavioral tracking, which both demonstrated consistent neuronal activity upon repeated stimulation. Finally, the neuroprotective potential of optogenetic collicular stimulation was investigated in mice of either sex subjected to a glaucoma model and a 63\% reduction in RGC loss was found. This work describes a new paradigm for optogenetic collicular stimulation and a first demonstration that increasing target neuron activity can increase survival of the projecting neurons.

Key words: neuroprotection; optogenetics; retina; retinal ganglion cell; superior colliculus

\section{Significance Statement}

Despite glaucoma being a leading cause of blindness and visual impairment worldwide, no curative therapies exist. This study describes a novel paradigm to reduce retinal ganglion cell (RGC) degeneration underlying glaucoma. Building on previous observations that RGC survival is supported by the target neurons to which they project and using an innovative optogenetic approach, we increased neuronal activity in the mouse superior colliculus, a main projection target of rodent RGCs. This proved to be efficient in reducing RGC loss in a glaucoma model. Our findings establish a new optogenetic paradigm for target stimulation and encourage further exploration of the molecular signaling pathways mediating retrograde neuroprotective communication.

\section{Introduction}

Glaucoma is a group of age-related neurodegenerative eye diseases characterized by a progressive degeneration of retinal gan-

Received April 6, 2018; revised Nov. 15, 2018; accepted Dec. 29, 2018

Author contributions: E.G. wrote the first draft of the paper; E.G., M.C., E.D., M.S.-N., L.D.G., C.V.d.H., I.S., V.B., L.A., and L.M. edited the paper; E.G., E.D., L.D.G., V.B., L.A., and L.M. designed research; E.G., M.C., M.S.-N., C.V.d.H., and I.S. performed research; C.V.d.H. contributed unpublished reagents/analytic tools; E.G. and M.C. analyzed data; E.G., L.A., and L.M. wrote the paper.

This work was supported by the Hercules Foundation (Grants AKUL-09-038 and AKUL1309); the Research Foundation Flanders (fellowships to M.C., E.D., M.S.-N., and L.D.G.); the Flemish government agency for Innovation by Science and Technology (fellowships to L.D.G., I.S., and E.G. and SBO projects 110068 OPTOBRAIN, 130065 MIRIAD and S006617N Neuro-TRAFFIC); and Funds for Research in Ophthalmology (E.G. and E.D.). We thank Samme Vreysen, Lut Noterdaeme, Marijke Christiaens, Evelien Herinckx, and Véronique Brouwers for excellent technical assis- glion cells (RGCs) and involves an early decrease in axonal transport, axon loss, dendritic remodeling, and finally apoptosis of the cell body (Calkins, 2012; Quigley, 2016; Jonas et al., 2017). As RGCs convey visual information from the eye to different visual centers in the brain, glaucomatous damage results in irreversible vision impairment and eventually culminates in blindness. Current therapies aim at lowering the intraocular eye

\footnotetext{
tance and Pieter Vancamp and all members of the OPTOBRAIN consortium for helpful comments regarding this manuscript and the experiments described therein.

The authors declare no competing financial interests.

Correspondence should be addressed to Lieve Moons at lieve.moons@kuleuven.be.

https://doi.org/10.1523/JNEUROSCI.0872-18.2018

Copyright $\odot 2019$ the authors $\quad 0270-6474 / 19 / 392313-13 \$ 15.00 / 0$
} 
pressure (IOP), the most recognized risk factor for glaucoma, and although this indeed reduces the risk of disease progression, visual impairment still occurs in a subset of patients (Heijl et al., 2002; Johnson et al., 2009; Heijl, 2013; Cohen and Pasquale, 2014). Therefore, new treatments to halt or prevent RGC degeneration are being actively investigated (Almasieh and Levin, 2017). One approach is ocular supplementation of neurotrophic factors, which are well known to promote neuronal survival, growth, and differentiation (Reichardt, 2006). Studies in different animal models of glaucoma repeatedly demonstrated that retinal neurotrophic supplementation results in a temporary neuroprotective effect, but falls short in promoting sustained RGC survival (Di Polo et al., 1998; Martin et al., 2003; Johnson et al., 2010; Dekeyster et al., 2015; Sánchez-Migallón et al., 2016). Alternatively, RGC survival can also be improved by retrograde neurotrophin supplementation from retinal brain target regions (Weber et al., 2010). Interestingly, it has been suggested that these target-derived neurotrophins can induce distinct signaling pathways compared with neurotrophins produced locally in the retina, highlighting this approach as a potential novel strategy for achieving long-term RGC protection (Matusica and Coulson, 2014; van Oterendorp et al., 2014). In previous work, we showed that continuous vector-based overexpression of brain-derived neurotrophic factor (BDNF) in the superior colliculus (SC), a midbrain structure known to receive projections of $85-90 \%$ of all murine RGCs, had no neuroprotective effect on RGCs in a mouse glaucoma model (Dekeyster et al., 2015; Ellis et al., 2016). We attributed this lack of efficacy to decreased neurotrophin responsiveness of the RGC terminals in the SC due to a parallel reduction in receptor expression. These findings argued for a different approach; instead of continuous overproduction of a single neurotrophic factor at (too) high levels, we hypothesized that controlled stimulation of neuronal activity in the SC might result in a balanced, physiologically relevant increase in a panel of molecules that may confer RGC neuroprotection upon retrograde transport. Indeed, the production of most neurotrophins is closely linked to neuronal activity (West et al., 2014). Specifically, both Bdnf and Ngf expression and release are well known to be upregulated by various activating stimuli such as electrical stimulation, excitatory neurotransmitters, and physiological activity of various brain regions (Zafra et al., 1990; Balkowiec and Katz, 2000; Hall et al., 2000; Li et al., 2000; Nanda and Mack, 2000; Rattiner et al., 2004).

Within this work, we sought to explore the potential of optogenetics for a controllable, prolonged induction of neuronal activity in SC and thereby enhance RGC survival in a mouse glaucoma model. To achieve protracted stimulation, we used the stabilized step function opsin (SSFO), a channelrhodopsin-2 (ChR2) mutant with a closing time constant of $\sim 30$ min (Yizhar et al., 2011). To maximize exposure of RGCs to target stimulation, the SSFO was introduced in the SC, a midbrain region to which most of the RGCs project (Ellis et al., 2016). Next, ocular hypertension and glaucomatous RGC death was induced via laser photocoagulation (LP) of the perilimbal and episcleral vessels of the mouse eye, a well established glaucoma model in rodents (Vidal-Sanz et al., 2012). Using this protocol, we here provide a proof of concept that increasing neuronal activity in the SC is neuroprotective for RGCs subjected to glaucomatous injury. Further investigation of the molecular signaling pathways mediating this neuroprotective effect could lead to the identification of novel therapeutic targets for glaucoma.

\section{Materials and Methods}

Mice. This study was conducted in compliance with the European Communities Council Directive of 22 September 2010 (2010/63/EU) and the Belgian legislation KB of 29 May 2013 and was approved by the KU Leuven institutional ethical committee (P193/2015). C57BL/6J mice (B6) of both sexes (JAX Mice Strain, stock \#000664, acquired via Charles River Laboratories, RRID:IMSR_JAX:000664) 2-3 months of age were obtained from the university breeding colony. All mice were kept under a 14/10 light/dark cycle with ad libitum access to food and water.

Viral vectors. To enable prolonged stimulation of the SC, two adenoassociated virus (AAV)-based vectors encoding the SSFO were tested: AAV2/1-CMV-intron-SSFO-mCherry (hereafter referred to as AAV2/1CMV-SSFO) and AAV2/7-CaMKII0.4-intron-SSFO-mCherry (AAV2/ 7-CaMKII-SSFO). The SSFO is the C128S/D156A mutant of ChR2 (sequence info at https://web.stanford.edu/group/dlab/optogenetics/ sequence_info.html\#ssfo) and was fused to a red fluorescent protein (RFP) reporter (mCherry) to allow easy visualization. All viral vectors were produced at the Leuven Viral Vector Core and vector titers were determined as genomic copies (GC) per milliliter as described previously (Van der Perren et al., 2011). The vectors were based on the AAV2 inverted terminal repeats and transpackaged in the AAV1 and AAV7 capsid, of which preliminary tests had shown these were suitable for SC transduction. The AAV2/1-CMV-SSFO vector, in which SSFO was under the control of the cytomegalovirus (CMV) promoter, had a genomic titer of $7.11 \mathrm{E}+11 \mathrm{GC} / \mathrm{ml}$. The AAV2/7-CaMKII-SSFO, containing a shortened $\mathrm{Ca}^{2+} /$ calmodulin-dependent protein kinase II (CaMKII) promoter, CaMKII0.4, had a titer of 1.62E + $12 \mathrm{GC} / \mathrm{ml}$ (Gerits et al., 2015; Scheyltjens et al., 2015). As a control vector, an AAV2/1 vector with CMV promotor driving expression of mCherry only (AAV2/1-CMV-mCherry, $9.82 \mathrm{E}+11 \mathrm{GC} / \mathrm{ml}$ ) was used.

Surgical procedures. Viral vectors were injected into the right SC. Mice were anesthetized with medetomidine and ketamine $(1 \mathrm{mg} / \mathrm{kg}$ body weight, Domitor, Pfizer and $75 \mathrm{mg} / \mathrm{kg}$, Anesketin, Eurovet). The scalp of the animal was shaved and local anesthetic was applied (xylocaine, lidocaine 5\%, AstraZeneca). A craniotomy was performed between bregma -3.60 and $-3.80 \mathrm{~mm}$ (lambda) and $0.5-0.7 \mathrm{~mm}$ to the right of the midline. For the injection, a glass capillary was mounted on a Nanoject II microinjector (Drummond Scientific) and inserted to a depth of $1.3 \mathrm{~mm}$ below the pia mater. The vector was infused at a rate of $100 \mathrm{nl} / \mathrm{min}$ for a total volume of $200 \mathrm{nl}$. After suturing and disinfecting the wound, anesthesia was reversed with $1 \mathrm{mg} / \mathrm{kg}$ atimapezol (Antisedan, Pfizer). Mice were given $5 \mathrm{mg} / \mathrm{kg}$ meloxicam subcutaneously (Metacam, BoehringerIngelheim) when necessary for postoperative pain relief.

To allow for optogenetic activation, an optic fiber with a diameter of $200 \mu \mathrm{m}$, contained in a metal casing with a magnet to facilitate coupling with a patch cord (MFC_200/245-0.37_1.9 mm_SMR_A60, Doric Lenses) was implanted through the cortex just above the right SC. At least 3 weeks after viral vector injection, mice were anesthetized with isoflurane (Iso-Vet $1000 \mathrm{mg} / \mathrm{g}$, Eurovet; 4\% for induction, $1-1.5 \%$ for maintenance). The skull was reexposed and a UV-curable layer (Optibond FL primer adhesive, Kerr) was applied to improve implant adhesion. The fiber tip was placed at a depth of $1.1 \mathrm{~mm}$ at the location of the viral vector injection. After filling the hole in the skull with elastomer (Kwik-cast, World Precision Instruments), the fiber casing was fixed to the skull using dental cement (Unifast TRAD, GC Europe). Finally, the skin was sutured and mice were given $5 \mathrm{mg} / \mathrm{kg}$ meloxicam subcutaneously (Metacam, Boehringer-Ingelheim) for postoperative pain relief.

The LP glaucoma model was performed as described previously (Valiente-Soriano et al., 2015; De Groef et al., 2016). Briefly, mice were anesthetized with a mixture of xylazine and ketamine $(10 \mathrm{mg} / \mathrm{kg}$ body weight, XYL-M 2\%, VMD Vetsupport, and $100 \mathrm{mg} / \mathrm{kg}$, Anesketin, Eurovet) and pupils were dilated with $1 \%$ tropicamide (Alcon). In a single session, a $532 \mathrm{~nm}$ laser (Vitra, Quantel Medical) was used to deliver $\sim 140$ spots to the perilimbal and episcleral vessels of the left eye. Laser power and duration were $100 \mathrm{~mW}$ and $0.05 \mathrm{~s}$, respectively. The right eye served as internal control. After the procedure, antibiotic ointment (tobramicine $3 \mathrm{mg} / \mathrm{g}$, Tobrex, Alcon) was applied to both eyes. 
IOP measurements. The IOP was measured with a calibrated rebound tonometer (Tono-lab, iCare) in awake mice as described previously (De Groef et al., 2016). IOP was assessed before LP and for 7 consecutive days following LP. Measurements were always made at 9:00 A.M. Ten independent IOP readings were collected per session, from which the two highest and lowest values were excluded to reduce variability. The mean of the remaining six values was taken as the IOP for that session.

Optogenetic stimulation. The implanted optic fiber was connected to a DPSS laser (473 nm, Shanghai Laser and Optics Century) using a patch cord, rotary joint, and optic fiber (Doric Lenses). Laser power was 2.2 $\mathrm{mW}$ except in a stimulation series with increasing light power, where the used powers are explicitly mentioned. Pulse duration was $2 \mathrm{~s}$ of continuous light, delivered using a custom-programmed Arduino chip. The optic fiber had a core diameter of $200 \mu \mathrm{m}$ with a numerical aperture of 0.37. Based on the online calculator https://web.stanford.edu/group/ $\mathrm{dlab} /$ cgi-bin/graph/chart.php, stimulation power of $2.2 \mathrm{~mW}$ led to a light power ranging from $39 \mathrm{o} 370 \mu \mathrm{W} / \mathrm{mm}^{2}$ at depths from 0.05 to $1 \mathrm{~mm}$. For the sham stimulation, mice were connected to the patch cord for $2 \mathrm{~s}$, but no light pulse was given. When performing the stimulation series, the same mice were repeatedly stimulated with increasing light power, with at least $2 \mathrm{~h}$ in between consecutive exposure to the increasing light pulses. For chronic stimulation, mice received a light pulse twice a day; the first stimulation was given at 10:00 A.M., the second at 5:00 P.M.

Behavioral testing. Mice were placed in a $25 \times 25 \mathrm{~cm}$ square arena and filmed at fixed distance from top view. Movies were recorded with a digital camcorder (DCR-SR37E, Sony) or an Ace GigE monochrome camera (acA1300-60 gm, Basler), recording at 24 frames/s. Animal tracking and analysis was performed with Ethovision XT 12 (Noldus) software. Animal movement rate was stratified into three predefined states with the Ethovision "mobility state" feature: highly mobile, representing running behavior $(>20 \%$ change in the detected location of the animal); mobile, representing walking (between $20 \%$ and $2 \%$ change); and immobile ( $<2 \%$ change), with all changes measured over an interval of four frames. Turning was quantified with the Ethovision "rotation" feature. A turn was defined as a center nose point rotation of 90 degrees with a threshold of 45 degrees in counterclockwise or clockwise direction. Behavioral trials consisted of tracking the baseline behavior of the animal for $30 \mathrm{~min}$, followed by light stimulation and tracking the resulting behavioral changes for another $30 \mathrm{~min}$. Data are presented as line graphs with mean \pm SEM at each time point unless otherwise indicated.

Tissue sampling. To reduce the normal visual activation of the SC and to limit non-optogenetically induced c-Fos expression, the final light or sham stimulation before tissue sampling was done after dark adaptation. Mice were placed in a dark room overnight and light or sham stimulated under dim red light. Next, 90 min after light or sham stimulation, mice were given an overdose of pentobarbital (Nembutal, CEVA Sante Animale) and transcardially perfused with saline and $4 \%$ paraformaldehyde (PFA) in PBS. Brains were dissected and fixed overnight at $4^{\circ} \mathrm{C}$ and eyes were enucleated and fixed for $1 \mathrm{~h}$ at room temperature, both in $4 \%$ PFA. Following dissection of the retina, the retinal flat-mounts were fixated again for $1 \mathrm{~h}$ in $4 \%$ PFA.

Processing of the retina. Retinal flat-mounts were immunostained for the RGC marker brain-specific homeobox/POU domain protein 3A (Brn3a), as described previously (Nadal-Nicolás et al., 2009). Briefly, retinas were frozen at $-80^{\circ} \mathrm{C}$ for $15 \mathrm{~min}$, followed by overnight incubation in PBS containing 2\% Triton X-100 (VWR), 2\% donkey serum, and goat anti-Brn3a (1/750, sc-31984, Santa-Cruz Biotechnologies, RRID: AB_2167511) at room temperature. Secondary antibody solution (donkey anti-goat Alexa Fluor 488, 1/500, Thermo Fisher) in PBS $+0.5 \%$ Triton X-100 was applied for $2 \mathrm{~h}$ at room temperature. Retinas were rinsed in PBS $+0.5 \%$ Triton X-100 in between steps and mounted with anti-fading mounting medium containing 10\% Mowiol 4-88 (SigmaAldrich), 40\% glycerol, and $0.1 \%$ 1,4-diazabicyclo-(2,2,2)-octane in 0.2 м Tris- $\mathrm{HCl}, \mathrm{pH} 8.5$ ).

A multiphoton laser confocal scanning microscope (FV1000-D, Olympus) was used to image the Brn3a-stained retinal flat-mounts. Mosaic images were created at with a $20 \times$ objective using Fluoview 4.2 software and exported as OIB files.
To quantify Brn3a-stained RGCs in flat-mounts, a previously described in-house ImageJ script was used (Geeraerts et al., 2016). Briefly, after noise removal steps, blob-like features are enhanced by creating an "eigenimage" based on the largest eigenvalues of the Hessian matrix. Then, RGCs are detected as local maxima, after which the user has the opportunity to outline the retinal flat-mount and exclude unwanted regions such as the optic nerve head and staining artifacts. Finally, the retinal surface area and RGC number are calculated, from which the RGC density can be derived. Results are presented in bar graphs with mean \pm SEM. To allow inspection of regional fluctuations in RGC density, the script also creates a cool-warm pseudocolored isodensity map, with retinal regions of high RGC density in yellow-white and regions with low density in cooler blueish hues.

Retrograde transduction of the retina following collicular injection of the AAV2/7-CaMKII-SSFO vector was investigated by checking the retina for RFP-positive RGCs. Retinal flat-mounts were double-stained for Brn3a and RFP by adding rabbit anti-RFP (1/5000, Rockland, RRID: AB_2209751) to the primary antibody mixture and donkey anti-rabbit Alexa Fluor 594 (1/200, Thermo Fisher) to the secondary antibody mixture of the Brn3a staining procedure described above. RFP-positive RGCs were manually counted in the entire retina using the Fiji cell counter plugin (RRID:SCR_002285) (Schindelin et al., 2012; Rueden et al., 2017). Results are shown as mean \pm SEM.

Processing of the SC. To evaluate changes in neuronal activity in the SC, immunostainings for the immediate-early gene transcription factor c-Fos were performed. Following antigen retrieval in citrate buffer (10 $\mathrm{mm}$ citrate, $0.05 \%$ Tween $20, \mathrm{pH} 6$ ) at $95^{\circ} \mathrm{C}$ for $20 \mathrm{~min}$, free-floating $50-\mu \mathrm{m}$-thick vibratome slices were incubated $20 \mathrm{~min}$ in $0.3 \% \mathrm{H}_{2} \mathrm{O}_{2}$ and incubated overnight with rabbit anti-c-Fos (1:7500 in PBS $+1 \%$ Triton $\mathrm{X}-100$, in-house produced and characterized) (Van Der Gucht et al., 2000). The following day, sections were incubated with goat anti-rabbit biotinylated secondary antibody (1/300 in PBS $+0.3 \%$ Triton X-100, DAKO) for $20 \mathrm{~min}$, followed by amplification and visualization using the TSA FITC system (PerkinElmer) according to the manufacturer's instructions. To investigate the spread of SSFO expression, SC slices were subjected to immunohistochemical staining for RFP. Free-floating 50 $\mu \mathrm{m}$ sections were incubated for $1 \mathrm{~h}$ in PBS $+0.1 \%$ Triton X-100 $+20 \%$ goat serum, followed by overnight incubation with rabbit anti-RFP (1/ 5000 in PBS $+0.1 \%$ Triton X-100, Rockland, RRID:AB_2209751). The next day, the secondary antibody (goat anti-rabbit Alexa Fluor 594; 1/200, Thermo Fisher) was applied for $2 \mathrm{~h}$. After both c-Fos and RFP staining, sections were incubated for $30 \mathrm{~min}$ with $4^{\prime}, 6$-diamidino-2phenylindole (DAPI) in PBS for nuclear counterstaining and mounted on glass slides with anti-fading mounting medium. Collicular sections were imaged using a $10 \times$ objective on a Zeiss Axio Imager Z.1 controlled via ZEN LE digital imaging software and exported in an open microscopy environment into a TIFF file.

Semiquantitative analysis of SSFO spread was assessed on coronal vibratome sections immunostained for the RFP reporter (see Fig. 1). A series of six slices interspersed $300 \mu \mathrm{m}$ from each other was found to sample most of the SC volume. For each section, the right SC was divided in four quadrants: medial superficial, lateral superficial, medial deep, and lateral deep, and expression was semiquantitatively graded from 0 (no SSFO expression) to 5 (complete transduction of quadrant area). The result of a section series was plotted along two "top views," one for the superficial and one for the deep SC, and these were averaged across mice to illustrate transduction throughout the SC. Results are shown as mean \pm SEM.

To investigate possible habituation effects after repeated optogenetic activation, a quantitative comparison of the density of c-Fos ${ }^{+}$cells per square millimeter of transduced tissue was performed for mice receiving single or triple stimulation. c-Fos counting was done on sections below the fiber and with the largest transduction area. Quantification was performed with an adapted version of the in-house ImageJ script used to count Brn $3 \mathrm{a}^{+}$RGCs in the retina. Results are shown as mean \pm SEM.

Experimental design and statistics. For histological results, each data point represents a sample (SC or retina) of one individual mouse, with the number of mice used indicated in the text and figure legends. For 


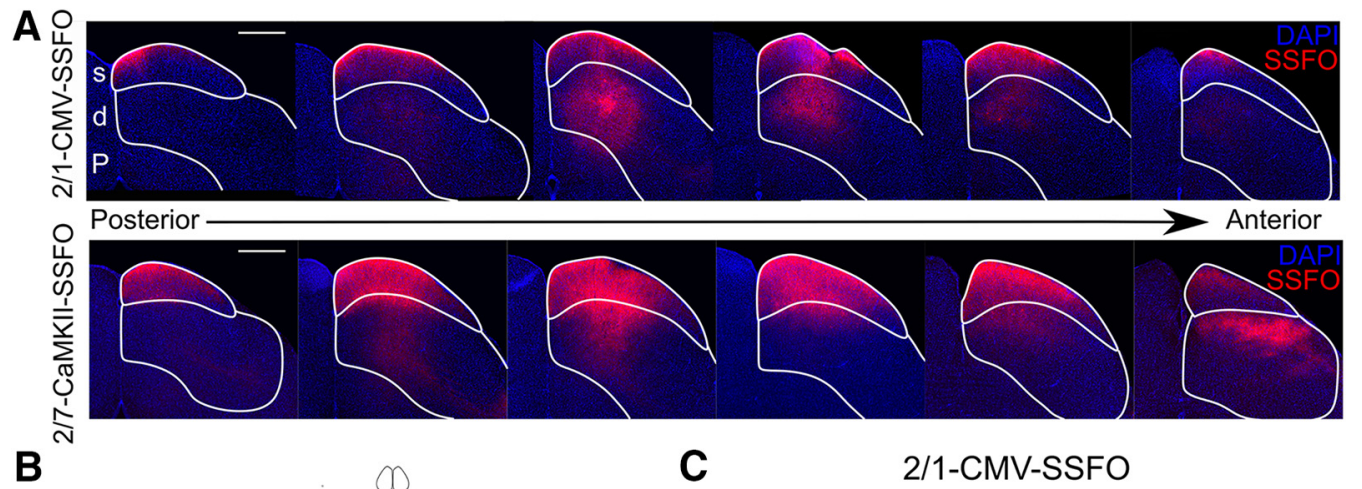

B

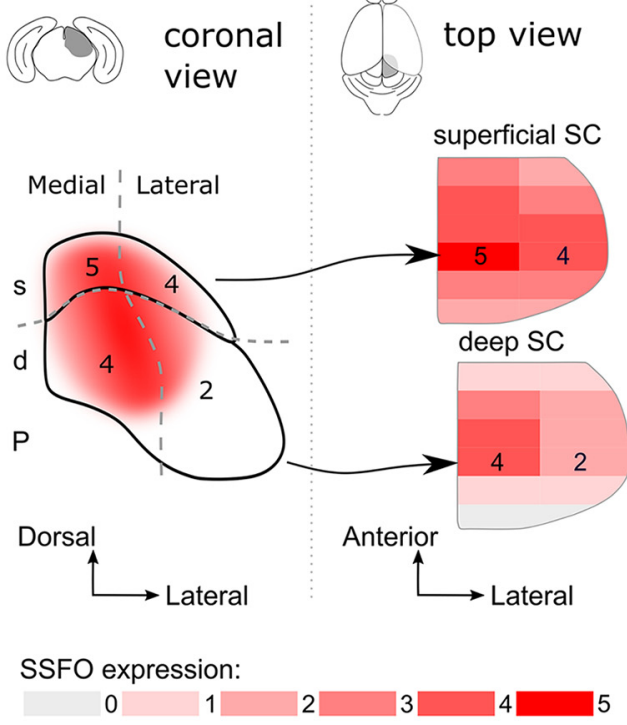

superficial SC

\begin{tabular}{|ll|ll|}
\hline $0.8 \pm 0.2$ & $0.8 \pm 0.2$ & deep SC & \\
\hline $2.1 \pm 0.3$ & $1.9 \pm 0.3$ & $0.8 \pm 0.2$ & $1.0 \pm 0.3$ \\
\hline $3.2 \pm 0.3$ & $2.7 \pm 0.3$ & $1.3 \pm 0.3$ & $1.0 \pm 0.3$ \\
$3.6 \pm 0.3$ & $3.2 \pm 0.3$ & $2.2 \pm 0.3$ & $1.1 \pm 0.2$ \\
$3.0 \pm 0.3$ & $3.0 \pm 0.3$ & $2.8 \pm 0.4$ & $1.9 \pm 0.2$ \\
\hline $2.6 \pm 0.4$ & $2.3 \pm 0.4$ & $1.9 \pm 0.4$ & $1.4 \pm 0.3$ \\
\hline & & $0.4 \pm 0.2$ & $0.3 \pm 0.2$ \\
\hline
\end{tabular}

D

\section{2/7-CaMKII-SSFO}

\begin{tabular}{|c|c|c|c|}
\hline \multicolumn{2}{|c|}{ superficial SC } & \multirow[b]{2}{*}{ eep SC } & \multirow[b]{3}{*}{$2.0 \pm 0.6$} \\
\hline $2.0 \pm 0.1$ & $1.8 \pm 0.3$ & & \\
\hline $2.9 \pm 0.3$ & $2.4 \pm 0.4$ & $2.3 \pm 0.5$ & \\
\hline $4.5 \pm 0.2$ & $3.8 \pm 0.3$ & $1.6 \pm 0.3$ & $1.3 \pm 0.3$ \\
\hline $4.9 \pm 0.1$ & $4.1 \pm 0.2$ & $3.1 \pm 0.4$ & $1.9 \pm 0.3$ \\
\hline $4.4 \pm 0.2$ & $4.0 \pm 0.3$ & $4.0 \pm 0.2$ & $2.8 \pm 0.3$ \\
\hline $3.9 \pm 0.1$ & $3.5 \pm 0.2$ & $3.3 \pm 0.4$ & $2.4 \pm 0.4$ \\
\hline & & $1.3 \pm 0.5$ & $0.8 \pm 0.4$ \\
\hline
\end{tabular}

Figure 1. Comparison of the AAV2/1-CMV-SSFO and AAV2/7-CaMKII-SSFO vector for transduction and activation of the SC. A, Transduction spread of viral vectors visualized by immunohistochemical staining for the SSF0-coupled RFP on vibratome sections throughout the SC spaced $300 \mu \mathrm{m}$ apart. The AAV2/7-CaMKII-SSF0 vector showed a more widespread transduction than the AAV2/1-CMV-SSF0 vector, especially in the superficial SC. An outline of the superficial SC and deep SC is sketched in white. Scale bar, $500 \mu \mathrm{m}$. $\boldsymbol{B}$, Theoretical example of semiquantitative analysis method to investigate SSF0 spread after viral vector injection. For each SC, six sections immunostained for the SSF0-fused RFP reporter were sampled at $300 \mu \mathrm{m}$ intervals, spanning most of the colliculus (similar to those shown in the first panel). In each section, transduction was assessed in four indicated SC quadrants (medial superficial, lateral superficial, medial deep and lateral deep) and graded on a scale from 0 to 5 , with 0 representing no and 5 representing complete transduction of the quadrant area. Results are plotted along top-view illustrations of the superficial and deep SC. These were averaged across mice to obtain an overview of SSFO transduction spread for a vector. The numbers depicted in this panel are only meant to illustrate the analysis; the results are shown in $\mathbf{C}$ and $\mathbf{D}$. C, D, Top view illustration of SC transduction by both vectors created as illustrated in $\boldsymbol{B}$. The resulting top view-like images are displayed, with average transduction \pm SEM for each position $(n=8-12)$. Both vectors showed good anterior-posterior and lateral medial spread in the superficial SC, with a more limited expression in the deep SC. The AAV2/7-CaMKII-SSF0 vector generally transduced more of the SC than the AAV2/1-CMV-SSFO vector. S, superficial SC; d, deep SC; P, periaqueductal gray.

results describing animal behavior upon optogenetic stimulation, the number of mice subjected to each protocol is stated.

Statistical analyses were performed with GraphPad Prism 7 software. Normality and equality of variance were confirmed with the D'AgostinoPearson omnibus K2 test and $F$ test, respectively. To investigate whether repeated collicular activation led to a reduced density of c-Fos ${ }^{+}$neurons compared with a single stimulation, a one-tailed unpaired $t$ test with $\left(n_{1}+n_{2}-2\right)$ degrees of freedom was used. When analyzing the IOP elevation after LP for optogenetically and sham-stimulated mice, a twoway ANOVA was used, with Dunnett's multiple-comparisons test to assess whether there was a significant difference with the LP + sham baseline condition. RGC density between optogenetically stimulated and sham mice was compared with a two-tailed unpaired $t$ test with $\left(n_{1}+n_{2}-2\right)$ degrees of freedom. To test whether optogenetic stimulation reduces the occurrence of sectorial loss in the LP model, a one-side Fisher's exact test was used. For correlation analysis of the number of RFP-positive RGCs versus RGC survival, a monotonic rather than a specifically linear relation between variables was assumed, so a Spearman's nonparametric correlation was used. A prob- ability level of $<0.05$ was considered statistically significant, with $p<0.05$ indicated in the figures as single asterisks.

\section{Results}

Expression and stimulation of the SSFO in the SC

The transduction efficiency of the AAV2/1-CMV-SSFO and AAV2/7-CaMKII-SSFO was assessed via immunohistochemical stainings for the RFP fusion protein on coronal sections spanning the entire SC (Fig. 1A). A single $200 \mathrm{nl}$ injection in the right SC at a depth of $1.3 \mathrm{~mm}$ turned out to be sufficient to induce SSFO expression throughout the injected colliculus. Although a complete anterior-posterior spread in the superficial layers of the SC was observed for both vectors, the AAV2/7-CaMKII-SSFO vector consistently showed a broader mediolateral spreading in the superficial SC at all bregma levels compared with the AAV2/1CMV-SSFO construct. In the deep SC, transduction was mostly 
A

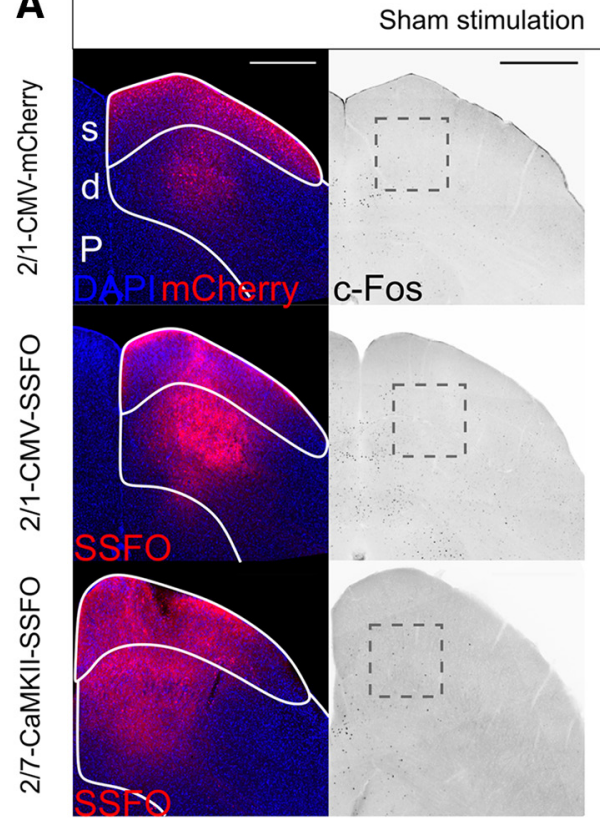

B

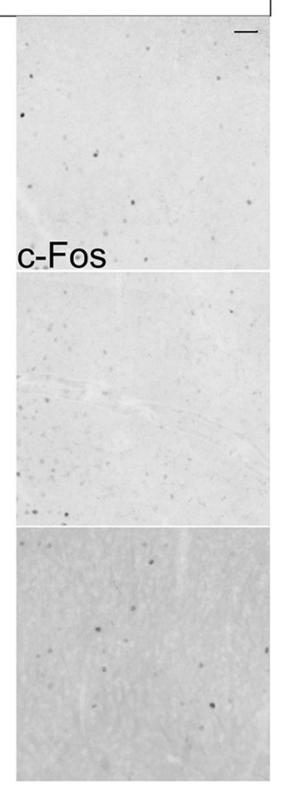

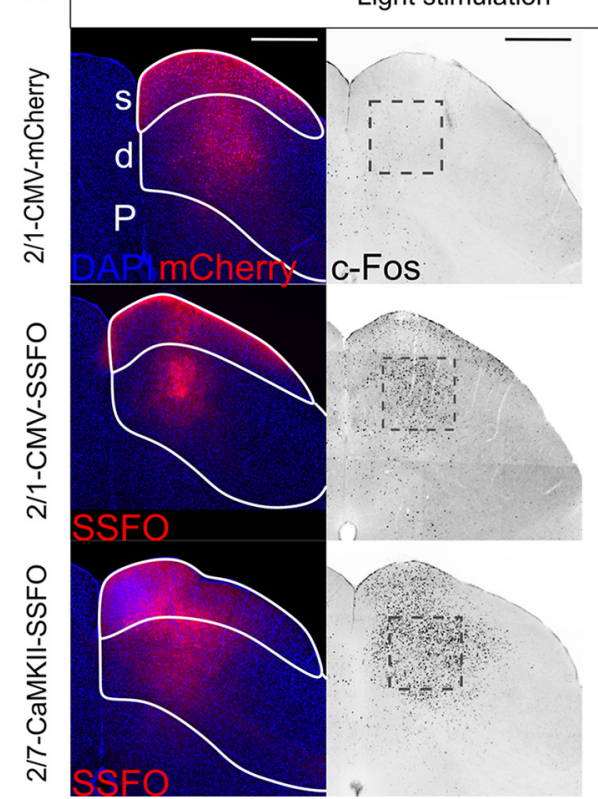

Light stimulation

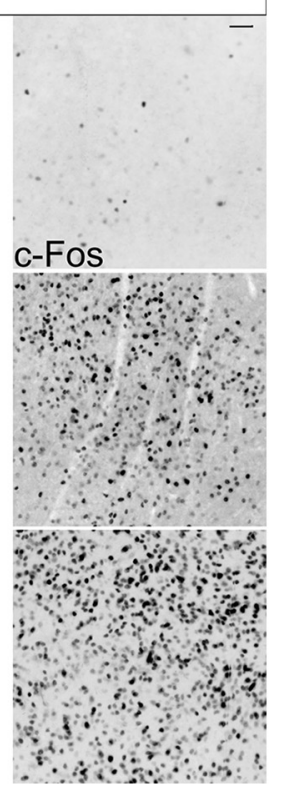

Figure 2. Optogenetic stimulation of the SSFO-transduced SC resulted in neuronal activation. $A$, Immunostaining for c-Fos on coronal vibratome sections revealed that sham stimulation does not result in c-Fos upregulation. $\boldsymbol{B}$, Light stimulation after injection of a mCherry control vector also failed to induce increased c-Fos expression, in contrast to light stimulation of a SSFO-transduced SC, in which c-Fos was strongly upregulated in the SSF0-expressing region. This indicates that both AAV2/1-CMV-SSF0 and AAV2/7-CaMKII-SSF0 are able to mediate optogenetic neuronal activation. $n=4$ for all conditions. Scale bar, $500 \mu \mathrm{m}, 50 \mu \mathrm{m}$ for magnified panels.S, superficial SC; d, deep SC; P, periaqueductal gray.

confined to the medial area around the injection site, thus showing a much more limited anterior-posterior spread for both vectors. Occasionally, transduction of the underlying periaqueductal gray was observed as well. Semiquantitative assessment of the collicular transduction patterns substantiated these qualitative observations, confirming the most widespread transduction for the AAV2/7-CaMKII-SSFO vector (Fig. $1 C, D$ ).

Next, immunostainings for the immediate-early gene c-Fos were used to assess optogenetic activation of collicular neurons expressing the SSFO (Fig. 2) (Van Der Gucht et al., 2000; Yizhar et al., 2011). Mice were subjected to overnight dark adaptation and stimulated in dim red light to exclude any visually driven activation of neurons in the SC. Any upregulation of c-Fos could therefore reliably be interpreted as the result of the optogenetic manipulation. A stimulation of $2 \mathrm{~s}$ with $2.2 \mathrm{~mW} 473 \mathrm{~nm}$ blue light was applied in these initial experiments (Scheyltjens et al., 2018). The experimental procedures of vector injection, optic fiber implantation, and light stimulation alone did not induce c-Fos expression in the SC. Mice injected with an mCherry-only control vector showed no c-Fos expression above baseline regardless of whether they received a single light pulse or a sham stimulation ( $n=4$ for both light and sham stimulation). Only mice injected with either of the SSFO-encoding vectors, AAV2/1-CMV-SSFO or AAV2/7-CaMKII-SSFO, showed strong induction of c-Fos upon a single light stimulation, but not upon sham stimulation ( $n=4$ for all groups). SSFO expression can thus specifically induce neuronal activation upon blue light activation. Comparison of c-Fos and SSFO expression on adjacent sections revealed that c-Fos expression was spatially constrained to the SSFOtransduced region of the SC (Fig. $2 B$ ).

In summary, whereas both AAV2/1-CMV-SSFO and AAV2/ 7-CaMKII-SSFO transduction allowed successful activation of neurons in the SC, the AAV2/7-CaMKII-SSFO vector was used in all further experiments to maximize transduction in the SC.

\section{Tunability of SSFO-mediated collicular stimulation}

Because the SC is involved in orienting or averting the body in response to sensory cues, a behavioral outcome of optogenetic activation of this brain structure is to be expected. Indeed, initial experiments of SSFO activation revealed a strong behavioral change characterized by abnormal running and turning. To determine whether this behavioral response could serve as a readout of successful collicular stimulation, a dose-response relation was established by stimulation with various light powers and quantifying the resulting behavioral response. Mice $(n=3)$ were injected with 2/7-CaMKII-SSFO and subjected to a stimulation series of $2 \mathrm{~s} 473 \mathrm{~nm}$ light with a power of $2.2 \mu \mathrm{W}$ up to $22 \mathrm{~mW}$, each time with a 10 -fold increment in light power and an interstimulus interval of $2 \mathrm{~h}$. A sham stimulation of $0 \mu \mathrm{W}$ with the laser turned off was included as well. The movement and turning behavior of the mice was automatically quantified using built-in features of Ethovision XT12 software (Fig. 3A). The movement rate was stratified into three types, immobile, mobile, and highly mobile, depending on the degree of body displacement by the animal per sample period of four frames. Rotation of the bodyhead vector of $>90$ degrees was counted as a turn (Fig. $3 B$ ). In all trials, the baseline behavior of mice was tracked in the stimulation cage for $30 \mathrm{~min}$ before stimulation for an internal control against which our main read-out, the behavioral change induced by optogenetic activation, could be compared.

Before stimulation, SSFO-expressing mice mostly explored the cage (mobile state) or paused to rest or groom (immobile state) and spent only limited time in a highly mobile state (Fig. $3 C-E)$. Mice that received a sham stimulation of $0 \mu \mathrm{W}$ continued this behavior for the full duration of the trial. Upon light stimulation with $220 \mu \mathrm{W}$ or more, the mice showed a brief undirected running response of $\sim 10 \mathrm{~s}$ (Fig. $3 E$ ). When mice were stimulated with $2.2 \mathrm{~mW}$, this undirected running after stimulation was fol- 
A

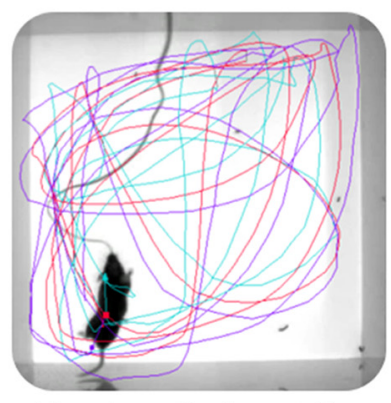

Head oBody oRear

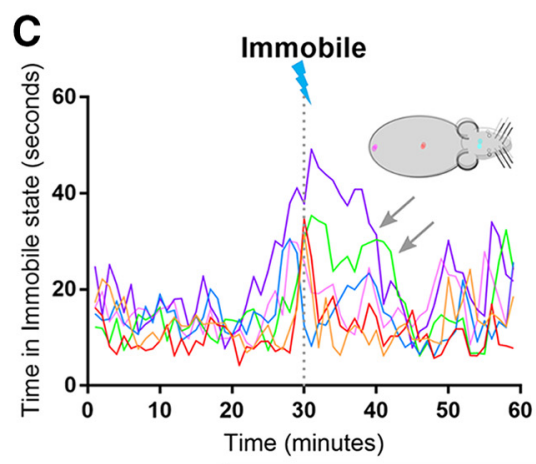

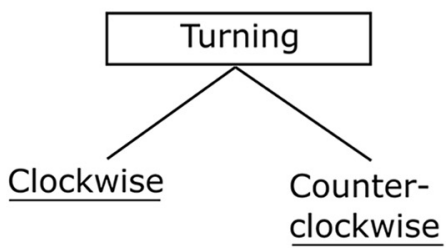

$90^{\circ}$ change of body-head vector in respective direction, with $45^{\circ}$ threshold
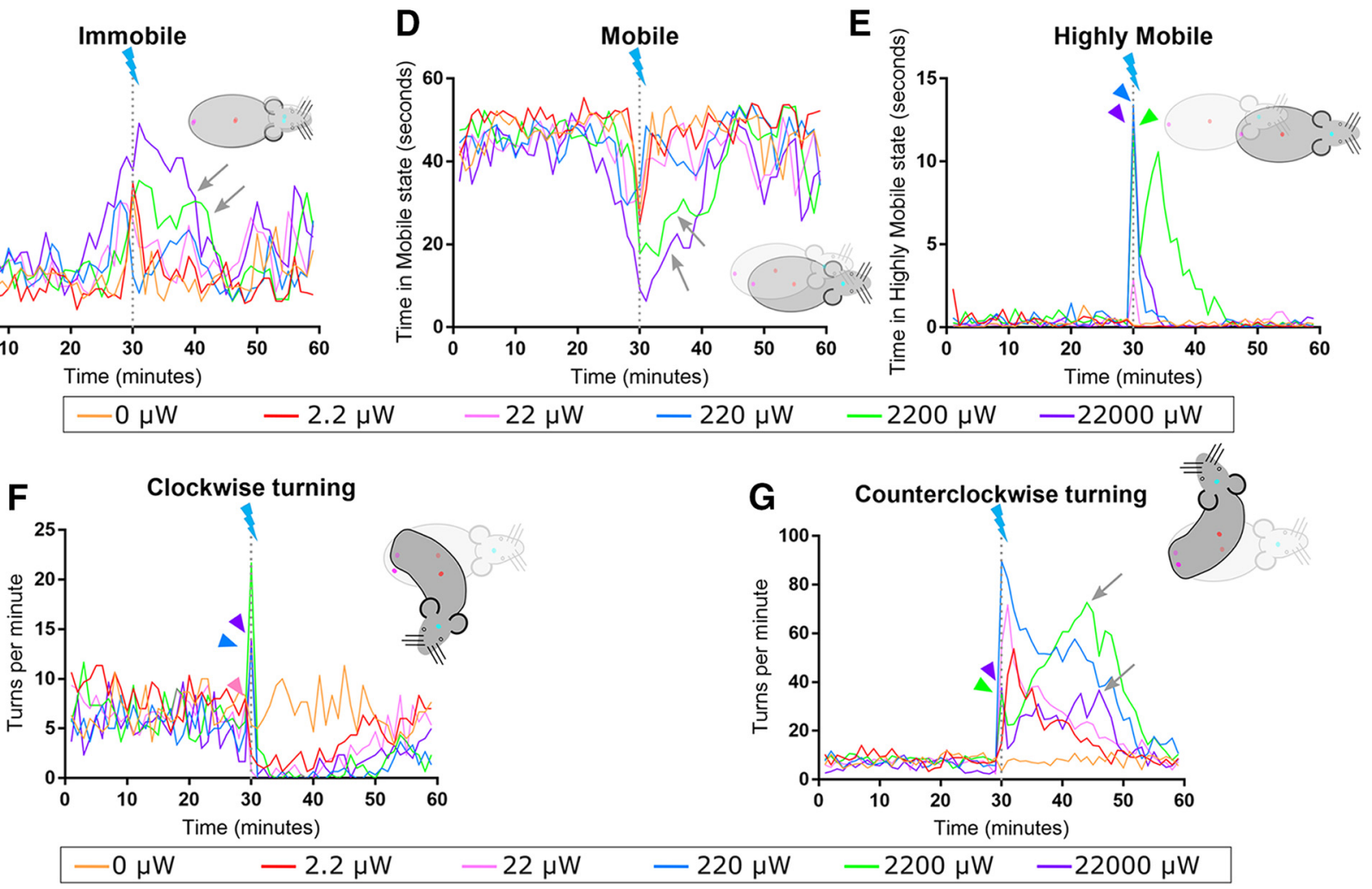

Figure 3. Behavioral tracking of AAV2/7-CaMKII-SSF0 transduced mice $(n=3)$ before, during, and after optogenetic stimulation with various light intensities. Animal behavior was recorded for $60 \mathrm{~min}$ and light stimulation $(2 \mathrm{~s}, 473 \mathrm{~nm})$ of specified light intensity was given at $30 \mathrm{~min}$, as marked on the graphs with a lightning bolt and vertical gray line. $A$, Example image of tracking software. The software tracked head, center, and tail base of the animal (in cyan, red, and purple, respectively). $\boldsymbol{B}$, Schematic overview of behavioral quantification parameters. Movement rate was stratified into three rates: immobile, mobile, and highly mobile, based on the body displacement over a four-frame interval. For each movement rate, a typical behavior is given. A turn was defined as a $90^{\circ}$ deviation of the body-head vector with a minimum threshold of $45^{\circ}$. C, Graph of mean time per minute in the immobile state for each stimulation intensity. Before stimulation, mice spent $10-20$ $\mathrm{s} / \mathrm{min}$ immobile due to resting or grooming. Right after stimulation, time in the immobile state increased for the stronger light powers for $\sim 10$ min (gray arrows), during which time the mice were highly alert and showed a high body tension (data not shown). $\boldsymbol{D}$, Graph depicting mean time per minute in the mobile state for each stimulation intensity. Before stimulation, mice spent $\sim 40 \mathrm{~s} / \mathrm{min}$ exploring the arena, moving at a normal, exploratory speed. After stimulation, this was reduced for the two highest stimulation powers for $\sim 10$ min (gray arrows). $\boldsymbol{E}$, Graph depicting mean time per minute in the highly mobile state for each stimulation intensity. Light powers of $220 \mu \mathrm{W}$ and more triggered a running response upon stimulation. $F, G$, Plots of the number of clockwise and counterclockwise turns, respectively. Before stimulation, mice had no preference for clockwise or counterclockwise movement. Stimulation induced a prominent increase in counterclockwise turning for up to $30 \mathrm{~min}$, with a concomitant decrease in clockwise turning. For stimulation powers up to $220 \mu \mathrm{W}$, the number of counterclockwise turns steadily decreased to baseline. For stimulations with $2.2 \mathrm{~mW}$, and to a lesser extent $22 \mathrm{~mW}$, the number of counterclockwise turns peaked $\sim 15 \mathrm{~min}$ after stimulation (gray arrows), when mobile behavior returned to baseline levels. All graphs show only the mean for each time point because error bars would compromise graph readability. Arrowheads point to peaks of their respective colors where the traces overlap.

lowed by an increase of immobile behavior interspersed with short bouts of running, which lasted for up to $15 \mathrm{~min}$ after stimulation. When stimulation power was increased to $22 \mathrm{~mW}$, this prolonged series of short runs was abolished and instead immobile behavior increased even further (Fig. 3C,D, gray arrows). When mice showed augmented immobile behavior after stimulation with 2.2 or $22 \mathrm{~mW}$, they were very alert with high body tension, suggesting that this immobility is a freezing-like state rather than a relaxed rest.
Turning behavior was also clearly affected by light stimulation (Fig. $3 F, G$ ). Mice performed $\sim 5-10$ turns in either direction per minute before stimulation and after sham stimulation. However, for all light powers used, even $2.2 \mu \mathrm{W}$, counterclockwise turning was notably increased up to $\sim 60$ turns per minute after stimulation. Clockwise turning was reduced during this timeframe, indicating a clear bias for counterclockwise turning. For stimulations with $2.2 \mathrm{~mW}$, and to a lesser extent $22 \mathrm{~mW}$, counterclockwise turning peaked when animal mobility returned to 
normal levels after a period of freezing-like behavior; that is, 10-15 min after the light pulse (Fig. 3G, gray arrows). This was not observed for weaker stimulations, which did not induce freezing-like immobility. There, the number of counterclockwise turns immediately peaked right after stimulation and gradually declined to baseline.

In summary, SSFO-mediated optogenetic stimulation of the right SC triggered increased and biased counterclockwise turning at lower light powers and this response was supplemented by running and freezing-like immobility at higher light powers. Furthermore, repeated light stimulation with a minimal interstimulus interval of $2 \mathrm{~h}$ still resulted in a mounting response to stimulation, arguing against a habituation effect. The duration of behavioral changes observed also matched well with the reported SSFO channel kinetics, arguing in favor of a prolonged stimulation of collicular activity for up to $30 \mathrm{~min}$ following a $2 \mathrm{~s}$ light pulse (Yizhar et al., 2011). Because specific behaviors were associated with light stimulation of a certain power, behavioral tracking could serve as confirmation of collicular stimulation in the following experiments.

\section{Consistency of repeated SSFO-mediated collicular stimulation}

With the stimulation power of $2.2 \mathrm{~mW}$ being the weakest stimulation driving the full spectrum of inducible behaviors (running, freezing-like immobility, and turning), this was chosen as the default stimulation power. To test the consistency of repeated SSFO-mediated stimulations and exclude any habituation effects, an experiment was set up in which three stimulations were spread over $5 \mathrm{~d}$ (Fig. 4A). All mice were injected with 2/7CaMKII-SSFO vector and light stimulated on days 1 and 3 with recording of the behavioral response. On day 5 , the mice either again received a light stimulation $(n=4)$ or were sham stimulated $(n=4)$ and the SC was then processed for evaluation of c-Fos expression.

Immunohistochemical staining at the endpoint showed that, for the mice receiving light stimulation for a third time at day 5 , c-Fos induction appeared similar in intensity and distribution to what was observed after a single stimulation (Fig. 4B). Quantification revealed that the density of c-Fos ${ }^{+}$cells in the transduced SC was not significantly lower for mice stimulated three times compared with a single stimulation $\left(2409 \pm 278\right.$ cells $/ \mathrm{mm}^{2}$ after single light stimulation vs $2649 \pm 218$ cells $/ \mathrm{mm}^{2}$ after 3 stimulations, $n=4$ for both groups, one-tailed unpaired $t$ test, $p=0.26$, degrees of freedom $=6$; Fig. $4 C$ ). For the mice receiving sham stimulation on day 5 , no such c-Fos upregulation was observed, confirming that the c-Fos expression at day 5 in light stimulated mice was not related to a previous stimulus. Therefore, neuronal activity in the $\mathrm{SC}$ returned to baseline between stimulations and the induced activation was consistent over multiple sessions, showing that there was no habituation to optogenetic stimulation.

In the behavioral trial on day 1 , animal mobility showed the same pattern as before, with mostly exploratory walking before stimulation and running upon stimulation, followed by $10 \mathrm{~min}$ of increased freezing-like immobility and returning to normal movement rate by 15 min after stimulation (Fig. $4 D$ ). For the turns, there was no preference before stimulation and an increase in turning to both sides during the running response, followed by increased and biased counterclockwise turning when mobility rate normalized. The second trial at day 3 revealed almost exactly the same pattern (Fig. 4D).
In summary, both histological and the behavioral read-out indicated that repeated optogenetic SC activation did not lead to habituation effects.

\section{Optogenetic stimulation is neuroprotective in the LP glaucoma model}

With consistent SSFO-mediated collicular stimulation established, this stimulation paradigm was applied in a mouse glaucoma model to test its neuroprotective potential (Fig. 5A). Following vector injection and optic fiber implantation, mice were subjected to the LP glaucoma model as described previously (Valiente-Soriano et al., 2015; De Groef et al., 2016). Starting 1 d before LP until tissue sampling at $14 \mathrm{~d}$ postinjury (dpi), the experimental group was light stimulated twice per day to induce chronic collicular activation (LP + stimulation group). The control group underwent identical viral vector injection, headpiece implantation, and LP induction, but was sham stimulated in parallel to the experimental group (LP + sham group).

The IOP profiles after LP of both groups indicated a similar induction of glaucomatous ocular hypertension regardless of optogenetic SC stimulation (two-way ANOVA, dpi: $p<0.0001$, $F_{(7,248)}=12.41$; stimulation: $p=0.37, F_{(1,248)}=0.80$; interaction: $\left.p=0.84, F_{(7,248)}=0.4832\right)($ Fig. $5 B)$. There was a significant IOP elevation for $\sim 5 \mathrm{~d}$, similar to previous reports using this model (Tukey's post hoc test, $p<0.05$ ) (Valiente-Soriano et al., 2015; De Groef et al., 2016). Habituation due to chronic stimulation could also be excluded for this LP + stimulation group. The effect on behavior of the second-to-last stimulation was recorded and quantified in a subset of chronically stimulated mice $(n=4)$ and indeed revealed that mobility and rotation patterns closely matched those of previous experiments (Fig. $5 C, D$ ). Corroborating these findings, $\mathrm{c}$-Fos expression patterns in the SC after the final stimulation also showed strong resemblance to those observed in nonglaucomatous mice stimulated one or three times, confirming that no habituation to repeated optogenetic stimulation occurred (Fig. 5E).

Next, RGC degeneration was assessed by immunohistochemical staining on retinal flat-mounts for the RGC nuclear marker Brn3a and semiautomatic quantification of the surviving RGC cell bodies at 14 dpi was performed (Fig. $5 F$ ). The LP + sham group showed an average survival of $2596 \pm 176 \mathrm{RGCs} / \mathrm{mm}^{2}$ in LP eyes contralateral to the stimulated SC $(n=20)$. This represented an average survival rate of $74 \%$ compared with contralateral eyes without LP. The LP + stimulation group had significantly more surviving RGCs: $3181 \pm 117 \mathrm{RGCs} / \mathrm{mm}^{2}$ corresponding to an average survival rate of $90 \%(n=17$, two-tailed unpaired $t$ test, $p=0.0114, t=2.67$, degrees of freedom $=35$ ). Because 929 RGCs $/ \mathrm{mm}^{2}$ were lost in the LP model at $14 \mathrm{dpi}$ compared with only $344 \mathrm{RGCs} / \mathrm{mm}^{2}$ after repeated optogenetic SC stimulation, RGC loss was reduced by $63 \%$. Isodensity maps of RGC survival for LP + sham eyes often showed the increased sectorial RGC loss associated with the model as blueish sectors, along with a general diffuse loss shown as orange-red hues (Fig. $5 G$ ). This sectorial loss occurred in 10/20 of the LP + sham eyes, whereas the other retinas just showed a diffuse loss. In contrast, in the LP + stimulation group, this sectorial loss occurred significantly less frequently, occurring in $3 / 17$ eyes, with the rest of the retinas showing diffuse loss (one-sided Fisher's exact test, $p=$ 0.04; Fig. 5G).

Finally, because retrograde transduction of RGCs could allow direct activation of transduced RGC terminals upon light stimulation in the SC, in contrast to the intended stimulation of the SC 
A

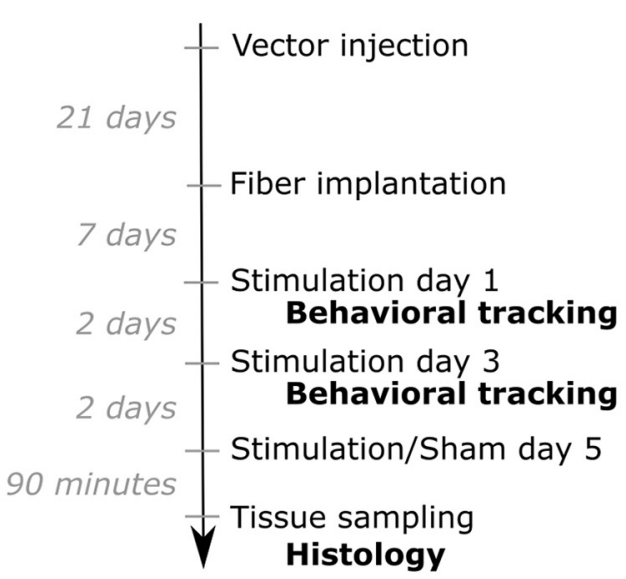

Histology
B

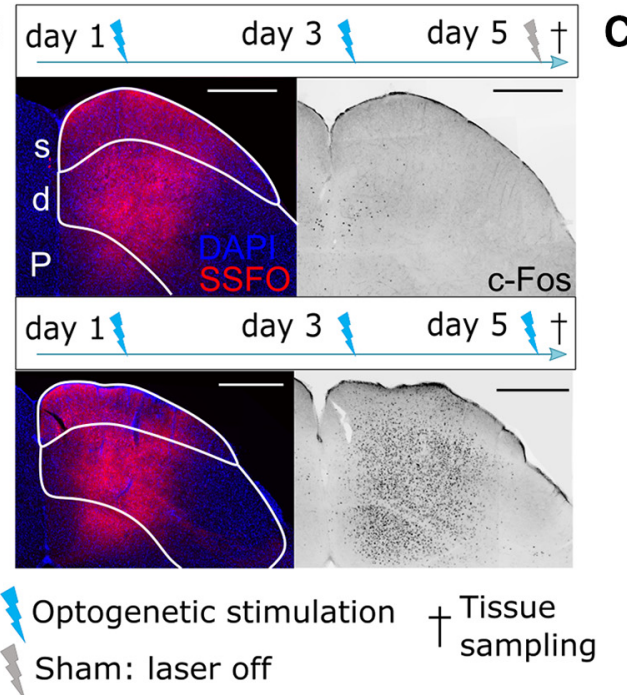

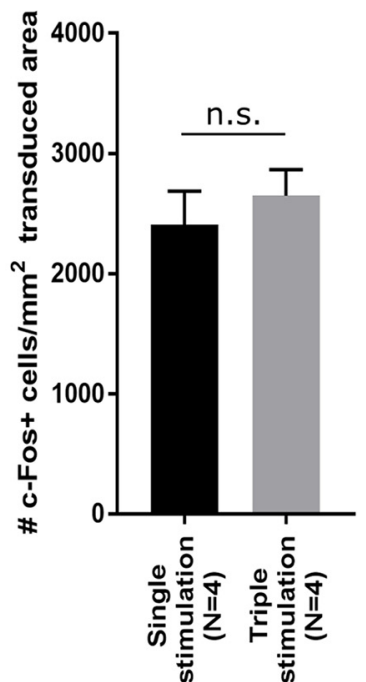
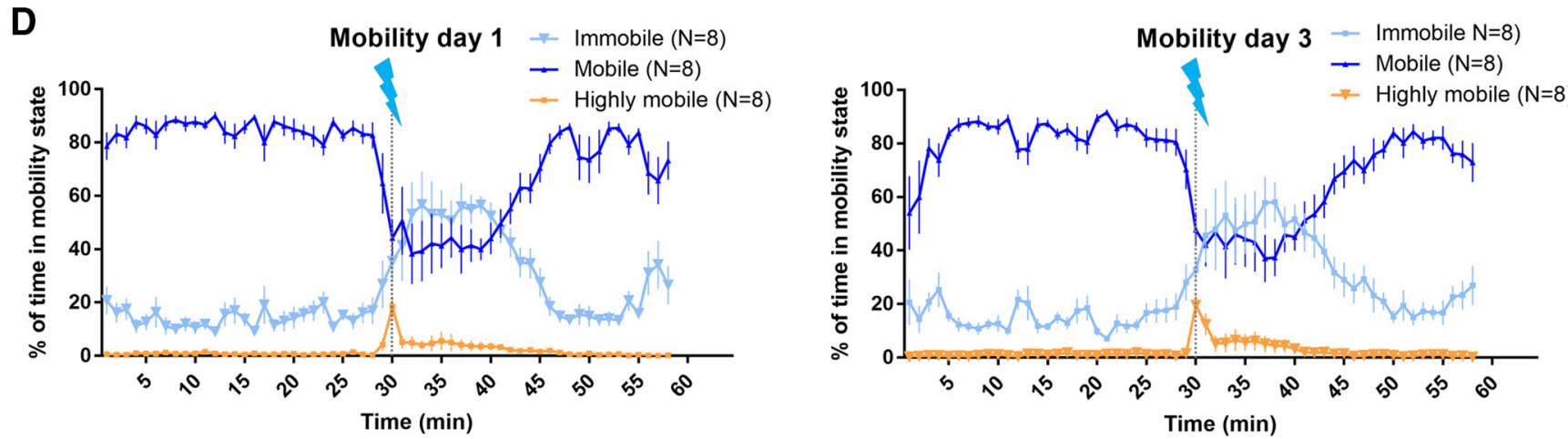

Turning day 1
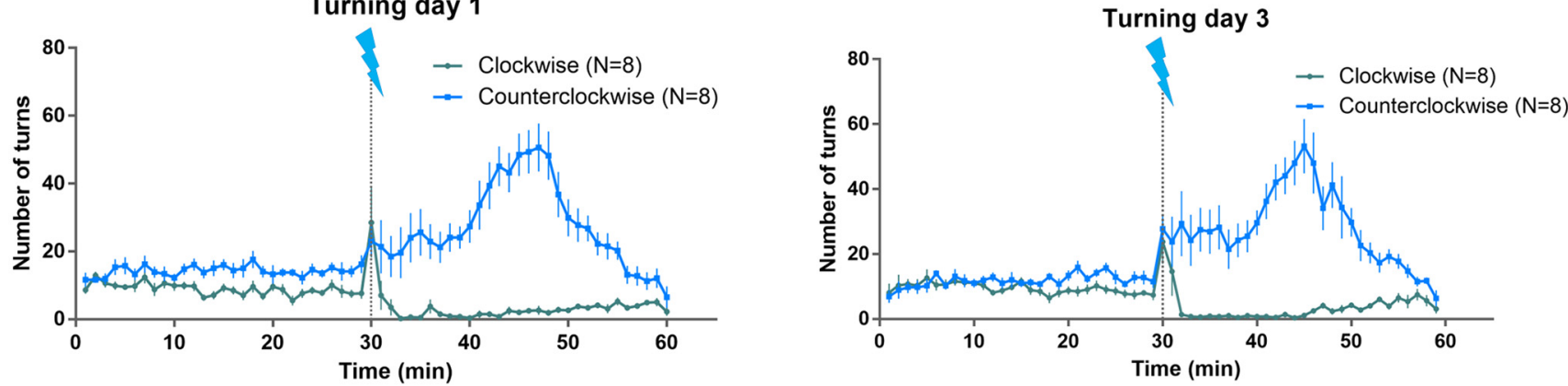

Figure 4. Repeated optogenetic stimulation of the $\mathrm{SC}$ results in a consistent immunohistochemical and behavioral response. Stimulation consisted of a $2 \mathrm{~s} 2.2 \mathrm{~mW} 473 \mathrm{~nm}$ light pulse and is indicated on graphs with a blue lightning bolt. A, Overview of experimental setup. All mice were injected with AAV2/7-CaMKII-SSF0 vector and light stimulated on day 1 and 3 with recording of the behavioral response. On day 5 , the mice either again received a light stimulation $(n=4)$ or were sham stimulated $(n=4)$ and the SC was then processed for evaluation of c-Fos expression. $\boldsymbol{B}$, The third stimulation on day 5 still elicited c-Fos activation in the transduced region, which was not the case if a sham stimulation was given on this final day ( $n=4$ for each group). Scale bars, $500 \mu \mathrm{m}$. C, Quantitative comparison of the density of c-Fos ${ }^{+}$cells in an SC section per millimeter of transduced tissue after single stimulation (Fig. 2B) or triple stimulation (Fig. 3B). There was no significant difference ( $n=4$ for both groups, on- tailed unpaired $t$ test, $p=0.26$, degrees of freedom $=6$ ). $\boldsymbol{D}$, SSFO-mediated optogenetic stimulation of the SC resulted in a prolonged behavioral response. Mice were tracked for $60 \mathrm{~min}$ and light stimulated at the $30 \mathrm{~min}$ mark, as indicated by gray dotted line with blue lightning bolt. Their response to the first optogenetic stimulation (day 1) was characterized by brief running upon light pulse, followed by increased immobility for $10 \mathrm{~min}$ and a preference for counterclockwise turning for up to 30 min. This response was highly similar in mice that were stimulated again $48 \mathrm{~h}$ later on day 3. S, superficial SC; d, deep SC; P, periaqueductal gray.

cells, possible confounding effects of retrograde SSFO transduction of RGCs in the retina had to be excluded. After immunostaining to enhance reporter visibility, we observed $49-371$ transduced RGCs/retina with a mean \pm SEM of $144 \pm 44$, representing $0.4 \%$ of the total RGC population $(n=7$, Fig. $5 H)$. Therefore, it seems unlikely that this limited population of SSFOexpressing RGCs could be responsible for the observed difference in RGC survival between stimulated and nonstimulated mice (585 RGCs $/ \mathrm{mm}^{2}$, corresponding to $\sim 7605 \mathrm{RGCs} /$ retina or $16 \%$ of the entire population). Importantly, further detailed evalua- tion disclosed that a higher number of retrogradely transduced RGCs did not result in an increased RGC survival; there was no correlation between the number of retrogradely transduced RGCs and RGC survival for light-stimulated mice (Spearman's $r=-0.57, p=0.2, n=7)$. The observation that maximally $2 \%$ of the protected RGCs is retrogradely transduced, together with the absence of a correlation between retrograde transduction and survival of RGCs, argues against a substantial role for such retrogradely transduced RGCs in the observed neuroprotective effect. 


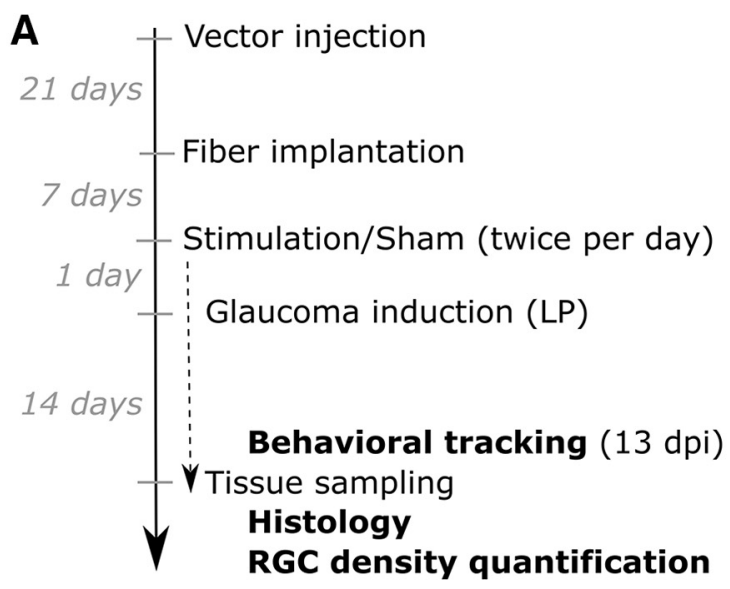

C

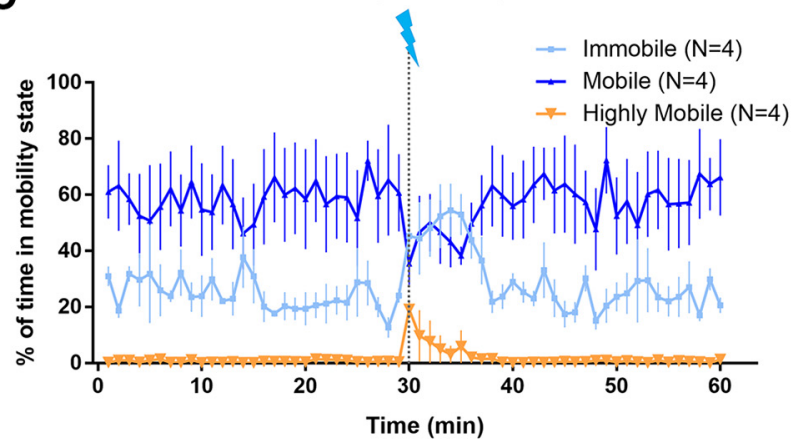

B

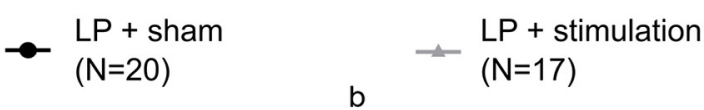
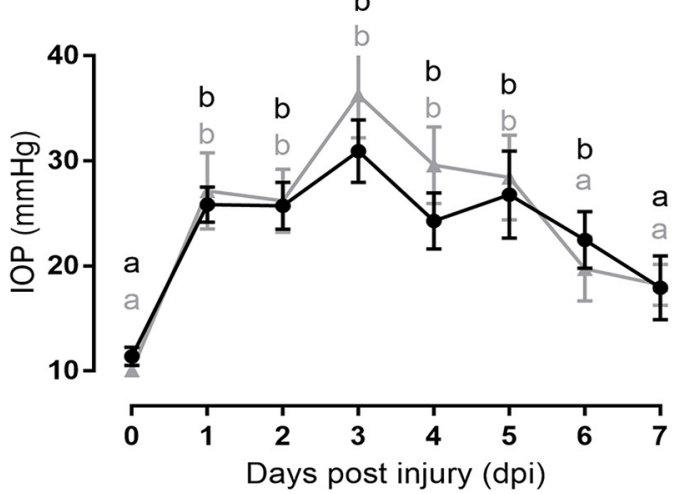

D

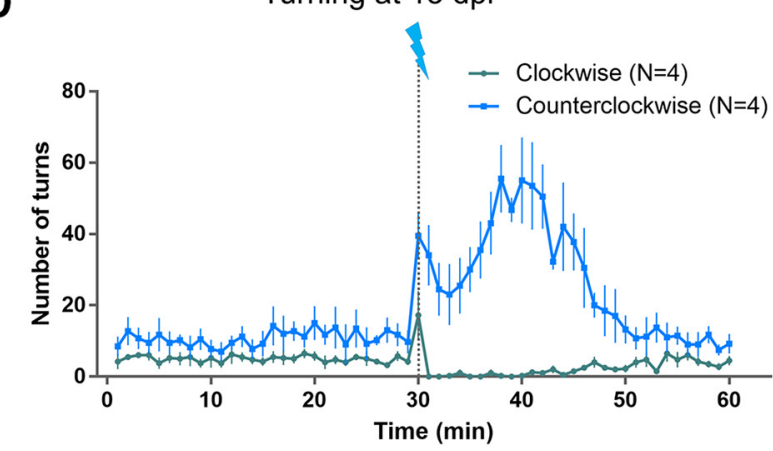

E

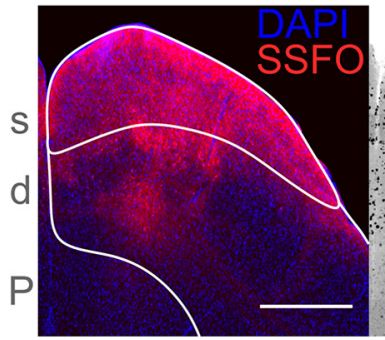

c-Fos expession at $14 \mathrm{dpi}$

F

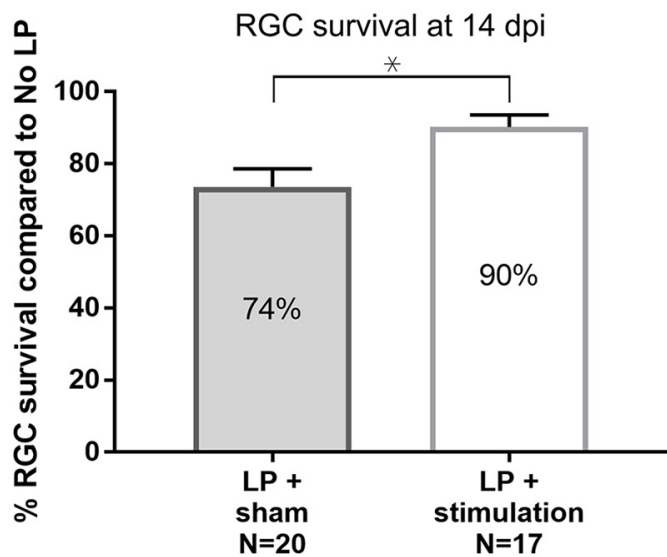

G No LP
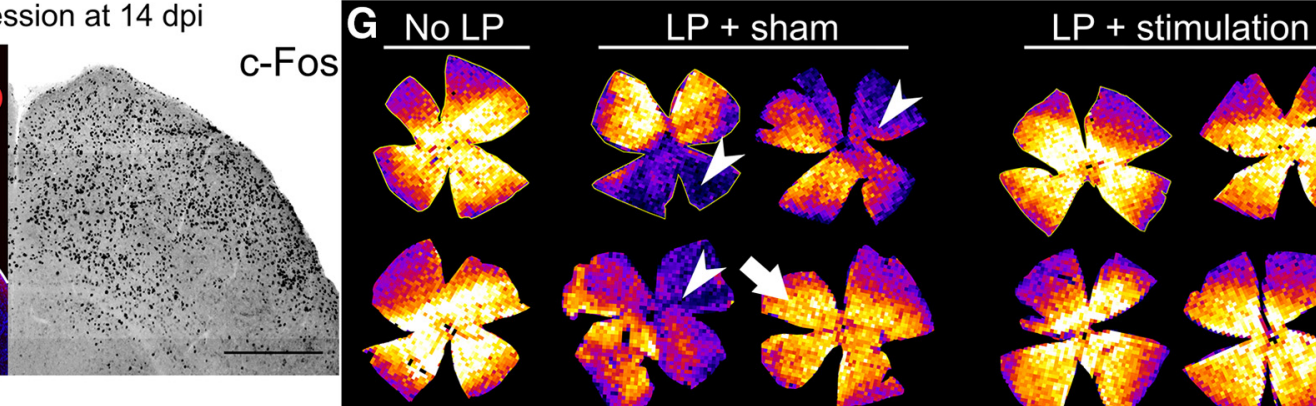
In summary, our findings indicate that the repeated optogenetic stimulation of neuronal activity in the SC confers retinal neuroprotection in a glaucomatous mouse model.

\section{Discussion}

In this study, we set out to determine whether target neuron stimulation can increase the survival of projecting neurons. Following intracollicular injection of an AAV2/7-CaMKII-SSFO vector, SSFO was expressed throughout the SC and could be repeatedly activated with consistent effects. Repeated optogenetic stimulation that increased neuronal activity of the SC was then shown to reduce RGC loss by $63 \%$ at 14 dpi in an LP mouse glaucoma model. Concomitantly, there was a significant reduction in the occurrence of sectorial cell loss, an important neurodegenerative aspect of the disease model (Valiente-Soriano et al., 2015).

Upon SSFO-mediated stimulation of the SC, a strong upregulation of c-Fos in the SSFO-transduced region of the SC was observed specifically after light delivery (Figs. $2 B, 4 B$ ). The immediate-early gene c-Fos is a well known transcription factor involved in coupling neuronal activity to translational changes and is widely used as a histological indicator of neuronal activation, also in the context of optogenetic neurostimulation (Curran and Morgan, 1995; Kovács, 1998; Yizhar et al., 2011). Behavioral investigation provided complementary information, unveiling that optogenetic activation recapitulated electrical and pharmacological stimulation of the SC by inducing a spectrum of running, freezing-like immobility, and turning responses (Kilpatrick et al., 1982; Schmitt et al., 1985; Sahibzada et al., 1986; Coimbra and Brandão, 1993; Brandão et al., 1994). In Sahibzada et al. (1986), directionality of turning was interpreted as orientation or aversive behavior, with orientation defined as a turn toward the visual hemifield governed by the stimulated SC, whereas aversion was marked by a turn in the opposite direction. In our study, the right SC was stimulated, so counterclockwise turns toward the left visual field can be interpreted as orientation behavior. Optogenetic stimulation of the right SC thus increases and biases orientation to the left hemifield. This is in partial agreement with Stubblefield et al. (2013), who reported a bias and no observation of direct movement by optogenetic stimulation alone, but this could be attributed to technical factors such as stimulation power and opsin. Apart from orientation behavior, defensive behaviors such as running and increased freezing-like immobility were also observed here, especially with higher stimulation powers. Our stimulation series of increasing light power suggests an interesting interplay between these defensive behaviors, with a stimulation of $2.2 \mathrm{~mW}$ eliciting bouts of running behavior intermixed with periods of increased freezing-like immobility, whereas in

\footnotetext{
$\leftarrow$

(Figure legend continued.) $\quad \boldsymbol{F}$, Repeated optogenetic stimulation of the SC led to an increased RGC survival in the LP glaucoma model at 14 dpi. Relative to contralateral right eyes, the LP + stimulation group showed significantly more survival $(90 \%, n=17)$ compared with the $\mathrm{LP}+\operatorname{sham}$ group $(74 \%, n=20)$ (two-tailed unpaired $t$ test, $p=0.0114, t=2.67$, degrees of freedom $=35)$. $G$, Representative isodensity maps of retinal flat-mounts. The no LP contralateral eye showed normal RGC density. LP + sham mice often had increased sectorial loss (arrowhead), although occasionally only diffuse loss was observed (arrow). For the LP + stimulation eyes, mostly diffuse loss was found. Scale bar, $2 \mathrm{~mm}$. $\boldsymbol{H}$, Limited retrograde transduction of the retina was observed after collicular vector injection. Panels in $\boldsymbol{H}$ show a representative part of the retina after double-staining for the RGC-marker Brn3a (green) and vector reporter RFP (black, artificial color for clarity). Retrogradely transduced RGCs are indicated with arrowheads and they represent $<1 \%$ of the total RGC population. Scale bar, $50 \mu \mathrm{m}$. S, superficial SC; d, deep SC; $P$, periaqueductal gray; ${ }^{*}$, significant difference with $P<0.05$.
}

sessions with a higher light power, this running was superseded by a further increase in immobile freezing-like behavior. Although previous reports have disclosed that optogenetic stimulation of various subpopulations of SC neurons can mediate defensive behaviors, we cannot exclude that direct activation of the periaqueductal gray, a known neuronal substrate of defensive behaviors, might have contributed to the observed responses (Carrive, 1993; Shang et al., 2015; Wei et al., 2015; Tovote et al., 2016). Indeed, despite considerable distance between the optic fiber positioned just above the SC and the periaqueductal gray under the deep SC, the excellent light sensitivity of the SSFO might allow direct stimulation of the periaqueductal gray with higher light power, as suggested by the augmented c-Fos expression seen in this region after a $2.2 \mathrm{~mW}$ light pulse (Fig. $4 B$ ) (Yizhar et al., 2011). In conclusion, the behavioral responses observed after SSFO-mediated stimulation of the SC recapitulate well known midbrain-related orientation and defensive behavior, thus confirming specificity of the stimulation paradigm used here (Dean et al., 1989; Brandão et al., 1994).

Both immunohistochemical and behavioral read-outs showed highly consistent results after repeated activation, suggesting a lack of habituation to the stimulation. The stimulation series with increasing light power resulted in a mounting behavioral response, indicating that a $2 \mathrm{~h}$ interstimulus interval was already enough to allow the response to reflect the stimulus parameters (Fig. 3). Furthermore, we demonstrated consistent c-Fos ${ }^{+}$cell densities and behavioral responses to repeated stimulations spread over $5 \mathrm{~d}$ (Fig. 4). When optogenetic stimulation was combined with the LP model and mice were stimulated twice per day for 2 weeks, we still observed a behavioral and histological response similar to mice stimulated for the first time (Fig. 5). These observations strongly argue against habituation, indicating that SSFO-mediated optogenetic activation presents a reliable, longterm stimulation strategy.

The most prominent finding of this work is that this repeated optogenetic stimulation of a major RGC target site, the SC, has a neuroprotective effect in the retina. Because we excluded a major contribution of the sparse RGCs that might have been directly activated due to SSFO expression in their axon terminals within the superficial layers of the SC, the mechanism of action is likely retrograde molecular signaling. Knowledge about retrograde signaling in RGCs is scarce and focused almost exclusively on neurotrophins such as BDNF. Importantly, the signaling pathways induced in RGCs by retrogradely derived BDNF have been shown to be distinct from those induced by BDNF supplied to the RGC soma in the retina, highlighting a key role for area-specific trophic stimulation (van Oterendorp et al., 2014; Dekeyster et al., 2015). During development, supplementation of BDNF to the SC reduces developmental RGC loss (Raff et al., 1993; Frade et al., 1997; Ma et al., 1998). Furthermore, retrograde neurotrophic factor deprivation is believed to be an important aspect of glaucomatous neurodegeneration, with a failure of retrograde axonal transport and spatially coincident increase of BDNF in collicular astrocytes (Nickells, 2007; Crish et al., 2013). Additionally, transient blocking of axonal transport using retrobulbar lidocaine injections rapidly reduces pattern electroretinogram amplitude, a measure of RGC function (Chou et al., 2013). These findings all suggest an important role for the SC in RGC function and survival and are now supplemented by an innovative and controllable approach of collicular stimulation with demonstrated neuroprotective effect.

A possible limitation of the current study is its reliance on loss of Brn3a expression to assess RGC survival because glaucoma- 
tous neurodegeneration has a much broader range of pathological features, including loss of functional anterograde and retrograde axonal transport, axon degeneration, and dendritic remodeling (Calkins, 2012). Here, we refrained from investigating axonal transport because the administration of fluorescent tracers entails a damaging manipulation of the retino-collicular axis, which might have acted as a confounder. Valiente-Soriano et al. (2015) describe a near-perfect linear correlation between survival of $\mathrm{Brn} \mathrm{a}^{+}$cells and preservation of retrograde axonal transport in the LP model applied here. Furthermore, compari-

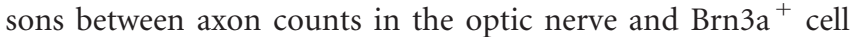
numbers are lacking for the LP model, but these measurements are in excellent correspondence in the microbead model, in which elevated IOP is achieved by intracameral injection of beads (Cone et al., 2010; Chen et al., 2011; Ito et al., 2016). Together, these results demonstrate that preservation of somatic Brn3a immunoreactivity is linked with other aspects of RGC integrity. There also has been some controversy on the validity of Brn3a as an RGC marker after injury because of its downregulation before actual RGC death (Nuschke et al., 2015; Mead and Tomarev, 2016). Although the loss of Brn3a immunoreactivity precedes clearing of the RGC soma by phagocytosing cells, discrepancies between both measurements are only temporary and progress toward the same endpoint, indicating that Brn3a is a sensitive predictor of RGC integrity (Salinas-Navarro et al., 2010; Galindo-Romero et al., 2011). Furthermore, previous studies clearly report that loss of Brn3a staining coincides with an increase in activated caspase- 3 expression after injury, indicating that the disappearance of Brn3a immunoreactivity signals the RGC entering apoptotic pathways (Sánchez-Migallón et al., 2016). Brn3a expression is also maintained by other known neuroprotective treatments such as intraocular BDNF administration (Sánchez-Migallón et al., 2011; Galindo-Romero et al., 2013). Therefore, although the present study does not provide a full quantification of all aspects of glaucomatous neurodegeneration, the use of Brn3a, an established marker for RGC survival with demonstrated correlation with other degenerative features, strongly argues in favor of an increased neuronal survival by target stimulation, with retrograde axonal signaling as the only known intermediate.

The SSFO-mediated collicular stimulation developed here might serve as a tool to unveil molecular signaling pathways by which target areas in the brain can support neuroprotection. Discovery of key molecular players underlying this survival signaling could provide new targets for future neuroprotective therapies. The eye and its connection to the central brain targets can potentially spearhead this search because, in mammals, it lacks the recurrent connections characterizing many other central brain regions. For example, in a rodent stroke model affecting the striatum and somatosensory cortex, optogenetic stimulation of the ipsilesional primary motor cortex was shown to promote functional recovery (Cheng et al., 2014). The intricate network of anterograde and retrograde connections between these brain structures, however, complicates interpretation of how optogenetic stimulation could mediate these effects (Ebrahimi et al., 1992; Petrof et al., 2015; Mohan et al., 2018). Therefore, the retino-collicular projection provides a unique opportunity to isolate the effect of retrograde signaling, in addition to the many advantages of the eye as a model system for neurodegenerative diseases (De Groef and Cordeiro, 2018). In summary, this work makes headway for the exploration of retrograde signaling as a route to novel neuroprotective therapies in the central nervous system.

\section{References}

Almasieh M, Levin LA (2017) Neuroprotection in glaucoma: animal models and clinical trials. Annu Rev Vis Sci 3:91-120.

Balkowiec A, Katz DM (2000) Activity-dependent release of endogenous brain-derived neurotrophic factor from primary sensory neurons detected by ELISA in situ. J Neurosci 20:7417-7423.

Brandão ML, Cardoso SH, Melo LL, Motta V, Coimbra NC (1994) Neural substrate of defensive behavior in the midbrain tectum. Neurosci Biobehav Rev 18:339-346.

Calkins DJ (2012) Critical pathogenic events underlying progression of neurodegeneration in glaucoma. Prog Retin Eye Res 31:702-719.

Carrive P (1993) The periaqueductal gray and defensive behavior: functional representation and neuronal organization. Behav Brain Res 58:27-47.

Chen H, Wei X, Cho KS, Chen G, Sappington R, Calkins DJ, Chen DF (2011) Optic neuropathy due to microbead-induced elevated intraocular pressure in the mouse. Invest Ophthalmol Vis Sci 52:36-44.

Cheng MY, Wang EH, Woodson WJ, Wang S, Sun G, Lee AG, Arac A, Fenno LE, Deisseroth K, Steinberg GK (2014) Optogenetic neuronal stimulation promotes functional recovery after stroke. Proc Natl Acad Sci U S A 111:12913-12918.

Chou TH, Park KK, Luo X, Porciatti V (2013) Retrograde signaling in the optic nerve is necessary for electrical responsiveness of retinal ganglion cells. Invest Ophthalmol Vis Sci 54:1236-1243.

Cohen LP, Pasquale LR (2014) Clinical characteristics and current treatment of glaucoma. Cold Spring Harb Perspect Med 4: a017236.

Coimbra NC, Brandão ML (1993) GABAergic nigro-collicular pathways modulate the defensive behaviour elicited by midbrain tectum stimulation. Behav Brain Res 59:131-139.

Cone FE, Gelman SE, Son JL, Pease ME, Quigley HA (2010) Differential susceptibility to experimental glaucoma among 3 mouse strains using bead and viscoelastic injection. Exp Eye Res 91:415-424.

Crish SD, Dapper JD, MacNamee SE, Balaram P, Sidorova TN, Lambert WS, Calkins DJ (2013) Failure of axonal transport induces a spatially coincident increase in astrocyte BDNF prior to synapse loss in a central target. Neuroscience 229:55-70.

Curran T, Morgan JI (1995) Fos: an immediate-early transcription factor in neurons. J Neurobiol 26:403-412.

Dean P, Redgrave P, Westby GW (1989) Event or emergency: 2 response systems in the mammalian superior colliculus. Trends Neurosci 12:137147.

De Groef L, Cordeiro MF (2018) Is the eye an extension of the brain in central nervous system disease? Journal of Ocular Pharmacology and Therapeutics 34:129-133.

De Groef L, Dekeyster E, Geeraerts E, Lefevere E, Stalmans I, Salinas-Navarro M, Moons L (2016) Differential visual system organization and susceptibility to experimental models of optic neuropathies in three commonly used mouse strains. Exp Eye Res 145:235-247.

Dekeyster E, Geeraerts E, Buyens T, Van den Haute C, Baekelandt V, De Groef L, Salinas-Navarro M, Moons L (2015) Tackling glaucoma from within the brain: an unfortunate interplay of BDNF and TrkB. PLoS One 10: e0142067.

Di Polo A, Aigner LJ, Dunn RJ, Bray GM, Aguayo AJ (1998) Prolonged delivery of brain-derived neurotrophic factor by adenovirus-infected muller cells temporarily rescues injured retinal ganglion cells. Proc Natl Acad Sci U S A 95:3978-3983.

Ebrahimi A, Pochet R, Roger M (1992) Topographical organization of the projections from physiologically identified areas of the motor cortex to the striatum in the rat. Neurosci Res 14:39-60.

Ellis EM, Gauvain G, Sivyer B, Murphy GJ (2016) Shared and distinct retinal input to the mouse superior colliculus and dorsal lateral geniculate nucleus. J Neurophysiol 116:602-610.

Frade JM, Bovolenta P, Martínez-Morales JR, Arribas A, Barbas JA, Rodriguez-Tebar A (1997) Control of early cell death by BDNF in the chick retina. Development 124:3313-3320.

Galindo-Romero C, Avilés-Trigueros M, Jiménez-López M, ValienteSoriano FJ, Salinas-Navarro M, Nadal-Nicolás F, Villegas-Pérez MP, Vidal-Sanz M, Agudo-Barriuso M (2011) Axotomy-induced retinal ganglion cell death in adult mice: quantitative and topographic time course analyses. Exp Eye Res 92:377-387.

Galindo-Romero C, Valiente-Soriano FJ, Jiménez-López M, García-Ayuso D, Villegas-Pérez MP, Vidal-Sanz M, Agudo-Barriuso M (2013) Effect of 
brain-derived neurotrophic factor on mouse axotomized retinal ganglion cells and phagocytic microglia. Invest Ophthalmol Vis Sci 54:974-985.

Geeraerts E, Dekeyster E, Gaublomme D, Salinas-Navarro M, De Groef L, Moons L (2016) A freely available semi-automated method for quantifying retinal ganglion cells in entire retinal flat-mounts. Exp Eye Res 147:105-113.

Gerits A, Vancraeyenest P, Vreysen S, Laramee ME, Michiels A, Gijsbers R, Van den Haute C, Moons L, Debyser Z, Baekelandt V, Arckens L, Vanduffel W (2015) Serotype-dependent transduction efficiencies of recombinant adeno-associated viral vectors in monkey neocortex. Neurophotonics 2: 031209.

Hall J, Thomas KL, Everitt BJ (2000) Rapid and selective induction of BDNF expression in the hippocampus during contextual learning. Nat Neurosci 3:533-535.

Heijl A (2013) The times they are a-changin': time to change glaucoma management. Acta Ophthalmol 91:92-99.

Heijl A, Leske MC, Bengtsson B, Hyman L, Bengtsson B, Hussein M; Early Manifest Glaucoma Trial Group (2002) Reduction of intraocular pressure and glaucoma progression: results from the early manifest glaucoma trial. Arch Ophthalmol 120:1268-1279.

Ito YA, Belforte N, Vargas JLC, Di Polo A (2016) A magnetic microbead occlusion model to induce ocular hypertension-dependent glaucoma in mice. J Vis Exp. Advance online publication. Retrieved March 23, 2016. doi: $10.3791 / 53731$

Johnson EC, Guo Y, Cepurna WO, Morrison JC (2009) Neurotrophin roles in retinal ganglion cell survival: lessons from rat glaucoma models. Exp Eye Res 88:808-815.

Johnson TV, Bull ND, Hunt DP, Marina N, Tomarev SI, Martin KR (2010) Neuroprotective effects of intravitreal mesenchymal stem cell transplantation in experimental glaucoma. Invest Ophthalmol Vis Sci 51:20512059.

Jonas JB, Aung T, Bourne RR, Bron AM, Ritch R, Panda-Jonas S (2017) Glaucoma. Lancet 390:2183-2193.

Kilpatrick IC, Collingridge GL, Starr MS (1982) Evidence for the participation of nigrotectal gamma-aminobutyrate-containing neurones in striatal and nigral-derived circling in the rat. Neuroscience 7:207-222.

Kovács KJ (1998) c-fos as a transcription factor: a stressful (re)view from a functional map. Neurochem Int 33:287-297.

Li XC, Jarvis ED, Alvarez-Borda B, Lim DA, Nottebohm F (2000) A relationship between behavior, neurotrophin expression, and new neuron survival. Proc Natl Acad Sci U S A 97:8584-8589.

Ma YT, Hsieh T, Forbes ME, Johnson JE, Frost DO (1998) BDNF injected into the superior colliculus reduces developmental retinal ganglion cell death. J Neurosci 18:2097-2107.

Martin KR, Quigley HA, Zack DJ, Levkovitch-Verbin H, Kielczewski J, Valenta D, Baumrind L, Pease ME, Klein RL, Hauswirth WW (2003) Gene therapy with brain-derived neurotrophic factor as a protection: retinal ganglion cells in a rat glaucoma model. Invest Ophthalmol Vis Sci 44: 4357-4365.

Matusica D, Coulson EJ (2014) Local versus long-range neurotrophin receptor signalling: endosomes are not just carriers for axonal transport. Semin Cell Dev Biol 31:57-63.

Mead B, Tomarev S (2016) Evaluating retinal ganglion cell loss and dysfunction. Exp Eye Res 151:96-106.

Mohan H, de Haan R, Mansvelder HD, de Kock CPJ (2018) The posterior parietal cortex as integrative hub for whisker sensorimotor information. Neuroscience 368:240-245.

Nadal-Nicolás FM, Jiménez-López M, Sobrado-Calvo P, Nieto-López L, Cánovas-Martínez I, Salinas-Navarro M, Vidal-Sanz M, Agudo M (2009) Brn3a as a marker of retinal ganglion cells: qualitative and quantitative time course studies in naive and optic nerve-injured retinas. Invest Ophthalmol Vis Sci 50:3860-3868.

Nanda SA, Mack KJ (2000) Seizures and sensory stimulation result in different patterns of brain derived neurotrophic factor protein expression in the barrel cortex and hippocampus. Mol Brain Res 78:1-14.

Nickells RW (2007) From ocular hypertension to ganglion cell death: a theoretical sequence of events leading to glaucoma. Can J Ophthalmol 42: 278-287.

Nuschke AC, Farrell SR, Levesque JM, Chauhan BC (2015) Assessment of retinal ganglion cell damage in glaucomatous optic neuropathy: axon transport, injury and soma loss. Exp Eye Res 141:111-124.

Petrof I, Viaene AN, Sherman SM (2015) Properties of the primary somato- sensory cortex projection to the primary motor cortex in the mouse. J Neurophysiol 113:2400-2407.

Quigley HA (2016) Understanding glaucomatous optic neuropathy: the synergy between clinical observation and investigation. Annu Rev Vis Sci 2:235-254.

Raff MC, Barres BA, Burne JF, Coles HS, Ishizaki Y, Jacobson MD (1993) Programmed cell-death and the control of cell-survival: lessons from the nervous-system. Science 262:695-700.

Rattiner LM, Davis M, French CT, Ressler KJ (2004) Brain-derived neurotrophic factor and tyrosine kinase receptor $\mathrm{B}$ involvement in amygdala-dependent fear conditioning. J Neurosci 24:4796-4806.

Reichardt LF (2006) Neurotrophin-regulated signalling pathways. Philos Trans R Soc Lond B Biol Sci 361:1545-1564.

Rueden CT, Schindelin J, Hiner MC, DeZonia BE, Walter AE, Arena ET, Eliceiri KW (2017) ImageJ2: ImageJ for the next generation of scientific image data. BMC Bioinformatics 18:529.

Sahibzada N, Dean P, Redgrave P (1986) Movements resembling orientation or avoidance elicited by electrical stimulation of the superior colliculus in rats. J Neurosci 6:723-733.

Salinas-Navarro M, Alarcón-Martínez L, Valiente-Soriano FJ, Jiménez-López M, Mayor-Torroglosa S, Avilés-Trigueros M, Villegas-Pérez MP, VidalSanz M (2010) Ocular hypertension impairs optic nerve axonal transport leading to progressive retinal ganglion cell degeneration. Exp Eye Res 90:168-183.

Sánchez-Migallón MC, Nadal-Nicolás FM, Jiménez-López M, SobradoCalvo P, Vidal-Sanz M, Agudo-Barriuso M (2011) Brain derived neurotrophic factor maintains Brn3a expression in axotomized rat retinal ganglion cells. Exp Eye Res 92:260-267.

Sánchez-Migallón MC, Valiente-Soriano FJ, Nadal-Nicolás FM, Vidal-Sanz M, Agudo-Barriuso M (2016) Apoptotic retinal ganglion cell death after optic nerve transection or crush in mice: delayed RGC loss with BDNF or a caspase 3 inhibitor. Invest Ophthalmol Vis Sci 57:81-93.

Scheyltjens I, Laramée ME, Van den Haute C, Gijsbers R, Debyser Z, Baekelandt V, Vreysen S, Arckens L (2015) Evaluation of the expression pattern of rAAV2/1,2/5, 2/7, 2/8, and 2/9 serotypes with different promoters in the mouse visual cortex. J Comp Neurol 523:2019-2042.

Scheyltjens I, Vreysen S, Van den Haute C, Sabanov V, Balschun D, Baekelandt V, Arckens L (2018) Transient and localized optogenetic activation of somatostatin-interneurons in mouse visual cortex abolishes long-term cortical plasticity due to vision loss. Brain Struct Funct 223: 2073-2095.

Schindelin J, Arganda-Carreras I, Frise E, Kaynig V, Longair M, Pietzsch T, Preibisch S, Rueden C, Saalfeld S, Schmid B, Tinevez JY, White DJ, Hartenstein V, Eliceiri K, Tomancak P, Cardona A (2012) Fiji: an opensource platform for biological-image analysis. Nat Methods 9:676-682.

Schmitt P, Di Scala G, Brandão ML, Karli P (1985) Behavioral effects of microinjections of SR 95103, a new GABA-A antagonist, into the medial hypothalamus or the mesencephalic central gray. Eur J Pharmacol 117: $149-158$

Shang C, Liu Z, Chen Z, Shi Y, Wang Q, Liu S, Li D, Cao P (2015) BRAIN CIRCUITS: a parvalbumin-positive excitatory visual pathway to trigger fear responses in mice. Science 348:1472-1477.

Stubblefield EA, Costabile JD, Felsen G (2013) Optogenetic investigation of the role of the superior colliculus in orienting movements. Behav Brain Res 255:55-63.

Tovote P, Esposito MS, Botta P, Chaudun F, Fadok JP, Markovic M, WolffSB, Ramakrishnan C, Fenno L, Deisseroth K, Herry C, Arber S, Lüthi A (2016) Midbrain circuits for defensive behaviour. Nature 534:206-212.

Valiente-Soriano FJ, Salinas-Navarro M, Jiménez-López M, AlarcónMartínez L, Ortín-Martínez A, Bernal-Garro JM, Avilés-Trigueros M, Agudo-Barriuso M, Villegas-Pérez MP, Vidal-Sanz M (2015) Effects of ocular hypertension in the visual system of pigmented mice. PLoS One 10:e0121134

Van Der Gucht E, Vandenbussche E, Orban GA, Vandesande F, Arckens L (2000) A new cat fos antibody to localize the immediate early gene c-fos in mammalian visual cortex after sensory stimulation. J Histochem Cytochem 48:671-684.

Van der Perren A, Toelen J, Carlon M, Van den Haute C, Coun F, Heeman B, Reumers V, Vandenberghe LH, Wilson JM, Debyser Z, Baekelandt V (2011) Efficient and stable transduction of dopaminergic neurons in rat substantia nigra by rAAV 2/1, 2/2, 2/5, 2/6.2, 2/7, 2/8 and 2/9. Gene Ther 18:517-527. 
van Oterendorp C, Sgouris S, Schallner N, Biermann J, Lagrèze WA (2014) Retrograde neurotrophic signaling in rat retinal ganglion cells is transmitted via the ERK5 but not the ERK1/2 pathway. Invest Ophthalmol Vis Sci 55:658-665.

Vidal-Sanz M, Salinas-Navarro M, Nadal-Nicolás FM, AlarcónMartínez L, Valiente-Soriano FJ, de Imperial JM, Avilés-Trigueros M, Agudo-Barriuso M, Villegas-Pérez MP (2012) Understanding glaucomatous damage: anatomical and functional data from ocular hypertensive rodent retinas. Prog Retin Eye Res 31:1-27.

Weber AJ, Viswanáthan S, Ramanathan C, Harman CD (2010) Combined application of BDNF to the eye and brain enhances ganglion cell survival and function in the cat after optic nerve injury. Invest Ophthalmol Vis Sci 51:327-334.

Wei P, Liu N, Zhang Z, Liu X, Tang Y, He X, Wu B, Zhou Z, Liu Y, Li J, Zhang Y, Zhou X, Xu L, Chen L, Bi G, Hu X, Xu F, Wang L (2015) Processing of visually evoked innate fear by a non-canonical thalamic pathway. Nat Commun 6:6756.

West AE, Pruunsild P, Timmusk T (2014) Neurotrophins: transcription and translation. In: Neurotrophic factors (Lewin GR, Carter BD, eds), pp 67-100. Berlin: Springer.

Yizhar O, Fenno LE, Prigge M, Schneider F, Davidson TJ, O’Shea DJ, Sohal VS, Goshen I, Finkelstein J, Paz JT, Stehfest K, Fudim R, Ramakrishnan C, Huguenard JR, Hegemann P, Deisseroth K (2011) Neocortical excitation/inhibition balance in information processing and social dysfunction. Nature 477:171-178

Zafra F, Hengerer B, Leibrock J, Thoenen H, Lindholm D (1990) Activity dependent regulation of bdnf and ngf messenger-rnas in the rat hippocampus is mediated by non-NMDA glutamate receptors. EMBO J 9:3545-3550. 